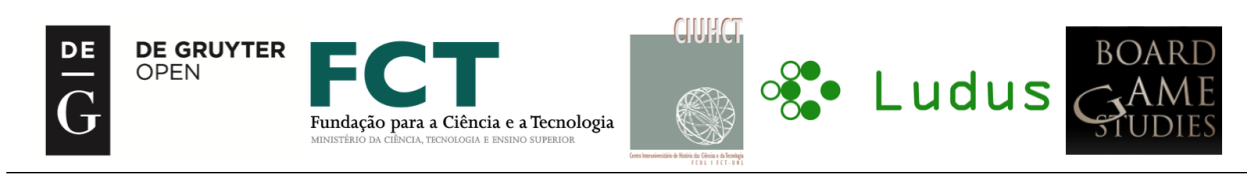

\title{
Die Kenntnis Des Dominospiels in Europa: Archäologie, Geschichte, BIBLIOGRAPHIE
}

\author{
Ingram Braun
}

\section{Zusammenfassung}

Die Geschichte des Dominospiels in Europa ist bisher wissenschaftlich nicht bearbeitet worden. Die ältesten Nachweise stammen aus China. Frühe archäologische Funde aus Nordwesteuropa reichen bis an die Grenze des Mittelalters zurück, sind aber außerordentlich selten. Ein Import über den Seeweg aus China kommt aus chronologischen Gründen nicht mehr in Betracht. Etwa ab 1760 gibt es schriftliche Belege aus Frankreich und Deutschland. Während sich aber in Frankreich darin ein Interesse der Oberschichten an wettkampfmäßigem Spiel manifestiert, handeln die deutschen Belege zunächst von einem Kinderspiel. Erst mit den militärischen Erfolgen Frankreichs um die Jahrhundertwende steigt die Reputation des Spiels in den europäischen Oberschichten. In dieser Zeit sind neben Spielsätzen aus Hartgeweben auch Kartenspielsätze geläufig. Der Name leitet sich vermutlich von dem französischen Wort für Buntpapierherstellung ab, unter dem auch die Kartenmacher zu subsumieren sind.

Schlüsselwörter: Domino, Spielbücher, Spielkarten, Europa, Neuzeit, Empire, Druckgrafik, Knochenschnitzerei

\section{Einleitung}

Der Fund eines Dominosteins bei Ausgrabungen in Dormagen-Stürzelberg (Rhein-Kreis Neuss, Nordrhein-Westfalen, Deutschland, s. Braun, o.J.) warf die Frage auf, seit wann Domino in Europa eigentlich nachweisbar ist? Überraschenderweise erweist es sich, dass es zu diesem Spiel keine systematische Untersuchung gibt. In wissenschaftlicher Literatur wird gelegentlich ein Zeitungsartikel von 1924 zitiert (Dubosc, 1927). Diesem Mangel soll hier abgeholfen werden.

Domino wird üblicherweise mit ziegelförmigen Spielsteinen gespielt, deren eine Seite an beiden Enden mit je einem Zahlenwert (meist in Form von

Board Game Studies Journal 10, pp. 61-100 DOI $10.1515 /$ bgs-2016-0004 
Tabelle 1: Umfang der Spielsätze $(0 \ldots n-1$ oder $1 \ldots n)$

\begin{tabular}{|c|c|c|c|c|c|c|c|c|}
\hline Verschiedene Werte $(n)$ & 6 & 7 & 8 & 9 & 10 & 11 & 12 & 13 \\
\hline Kombinationen (Steine) & 21 & 28 & 36 & 45 & 55 & 66 & 78 & 91 \\
\hline
\end{tabular}

Punktaugen wie beim Würfel) beschriftet ist. Es wird verdeckt gemischt und ausgeteilt, und dann dürfen die Spieler ihre Steine reihum an eines der beiden Enden der Kette aus bereits ausgespielten Steine dort anlegen, wo der einzelne Wert des alten und des neuen Steines übereinstimmen. Wer keinen passenden Wert hat, muss passen bzw. einen Stein ziehen, soweit noch welche übrig sind. Gewonnen hat, wer als erster alle Steine ablegen konnte. Wenn das niemandem gelingt, dann derjenige, der die niedrigste Augenzahl auf der Hand hat. Es gibt Spiele in verschiedenen Größen, wobei europäische Spiele üblicherweise jede Kombination genau ein einziges Mal aufweisen (Tab. 1).

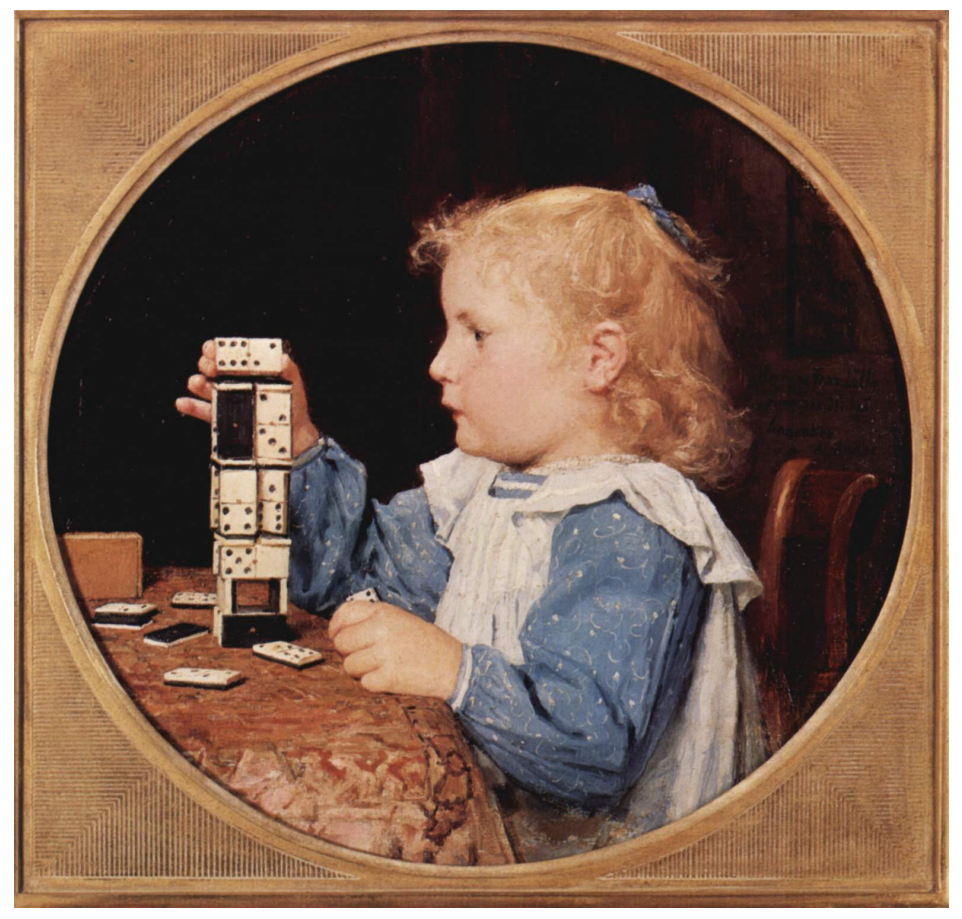

Abbildung 1: Albert Anker (1831-1910): Das Mädchen mit den Dominosteinen. Öl auf Holz, Durchmesser $37 \mathrm{~cm}$, Frankreich oder Schweiz, zweite Hälfte 19. Jahrhundert, Privatbesitz, Quelle: Wikimedia Commons. 
Mit Dominosteinen kann man auch Puzzleaufgaben lösen (van Delft und Botermans, 2004, S. 57-64) oder sie als Bauklötze verwenden (Abb. 1) 1 Man braucht auch nicht unbedingt Dominosteine dazu. Im Prinzip lässt es sich mit auch mit üblichen Spielkartensätzen spielen. Solche Kartendominos sind z. B. im angelsächsichen Raum als Stops bekannt, weil das Fehlen einer passenden Karte den Spieler stoppt (Morehead und Mott-Smith, 1983 , 91-96). Allerdings hat auch dort diese Gruppe keine Casino- oder Turnierspiele hervorgebracht, sondern nur einfache Spiele für den Familienkreis. Bei Kartenspielen geht einer der wesentlichen Merkmale des klassischen Domino verloren. Dort liegen nämlich alle ausgespielten Steine offen, und der Spieler kann bei jedem Zug alle verfügbaren Informationen eruieren. Bei Kartenspielen hingegen muss man sich in der Regel merken, welche Karten bereits ausgespielt wurden (N. N., 1829, 2. Abschnitt, S. 37).

\section{China}

Während man in Europa Spielkarten und ziegelförmige Spielsteine für Domino oder Mahjong (engl. tile games) terminologisch streng unterscheidet, werden in China beide Formen unter dem Begriff pai subsumiert (Lo, 2004, S. 217) Spielkarten existieren in China mindestens seit dem Ende des 13. Jahrhunderts und damit ein Jahrhundert früher als in Europa (Lo, 2000, S. 389). Der Autor Qu You, der von 1347 bis 1433 lebte, ist Verfasser des frühesten Dominohandbuches (Lo, 2000, S. 401). Es ist aber wahrscheinlich, dass bereits eine Quelle des 12.-13. Jahrhunderts das Spiel unter von Hausierern feilgebotenen Waren nennt. Nach Angaben in französischer Literatur sind bereits im 12. Jahrhundert in China beinerne Dominosteine nachgewiesen, die als Proto-Kartenspiel zu gelten haben (Lhôte, 1994, S. 461; Depaulis, 2007, S. 75). Allerdings gibt es keine gesicherten archäologischen Funde, sondern lediglich einen angeblich aus dem 12.-14. Jahundert stammenden Satz aus einer Privatsammlung, für den nicht einmal ein Fundort bekannt ist (Lo, 2004, S. 223 u. Abb. 17:7). Es werden sowohl Ein- als auch Mehrpersonenspiele beschrieben, wobei die Spielsätze meist aus 32 Steinen ohne Blankwert (1-6, davon elf doppelt) bestehen (Culin, 1895, S. 508-510) - es

\footnotetext{
${ }^{1}$ Dasselbe Motiv (Kind, das Dominosteine als Bauklötze verwendet), lediglich in Ganzkörperdarstellung, findet sich auch auf einem 1892 entstandenen Bild von Ellen M. Simpson in Privatbesitz. Im Metropolitan Museum New York befindet sich eine 1903 erworbenes Bronzerelief des Franzosen Alexandre Charpentier (1856-1909), das die Büste eines nackten Jungen zeigt, der wohl wirklich Domino spielt (Inv.-Nr. 03.7.14).

${ }^{2}$ Mahjong wird gerne als ,,chinesisches Domino“ bezeichnet. Aber es ist jung (19. Jahrhundert), wird mit Würfeln gespielt, und die Spieler versuchen, durch Ablegen und Ziehen von Steinen Figuren zu sammeln. Systematisch gehört es zur Rommé-Familie.
} 


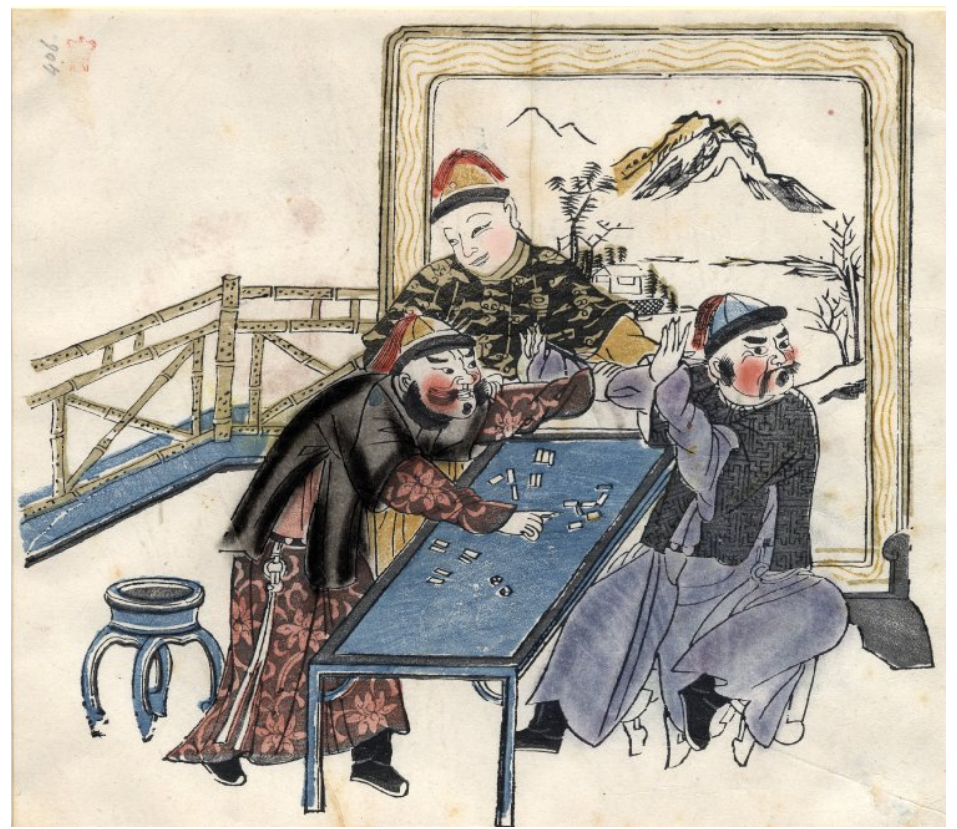

Abbildung 2: Chinesen beim Dominospiel. Holzschnitt, Farbdruck, China, 17. Jahrhundert, Britisches Museum London (Inv.-Nr. 1928,0323,0.35), Maße unbekannt.

gibt aber zahlreiche Varianten, auch mit Nullen (Lo, 2004, S. 223-224). Die missverständliche Mitteilung von Bell (1979, Bd. 1, S. 163), dass chinesische Spiele keine Nullen enthalten, beruht wohl auf der Annahme, das europäische Domino hätte sich unmittelbar aus dem chinesischen Standardspielsatz entwickelt. Tatsächlich ist in einem chinesischen Werk um 1600 ein 56teiliges Spiel mit Blanken beschrieben, das de facto eine Verdoppelung des europäischen Standardspielsatzes aus 28 Steinen darstellt (Lo, 2004, S. 224). Zudem erwähnt eine ethnologische Studie über Domino bei chinesischen Arbeitern in den USA mehrere ostasiatische Museumsstücke mit Blanken (Culin, 1895. S. 519-520). 1795 notiert ein niederländischer Kaufmann, der mehr als 15 Jahre in China verbracht hat, dass chinesisches Domino „exakt unserem entspricht und auch genauso gespielt wird" (Van Braam-Houckgeest, 1798, S. 342; 1799, S. 595). Auch die älteste Abbildung stammt aus China, ist aber leider nicht sehr detailliert (Abb. 2). Man kann nicht erkennen, ob mit Karten oder Steinen gespielt wird, da chinesische Spielkarten sehr viel schmaler sind als europäische. 


\section{Literarische Quellen des 18. und frühen 19. Jahrhun- derts}

Im 18. Jahrhundert wurden im westlichen Europa Spielanthologien, in denen die Regeln und Hinweise zu Strategie und Taktik versammelt waren, populär ${ }^{3}$ Diese Bücher dienten nicht bloßen Unterhaltungszwecken. Das weitverbreitete Spiel um Geld machte es notwendig, über einheitliche Regeln zu verfügen. Zudem dienten Verabredungen zum Spiel auch gesellschaftlichen Zwecken, um Konversationen anzubahnen. Dabei wollte man sich natürlich nicht durch Unkenntnis blamieren. Vorreiter im 17. Jahrhundert waren seit 1654 La Maison académique contenant un recueil général de tous les jeux divertissans pour se rejouyr agréablement dans les bonnes compagnies von D. H. la Marinière, das später unter dem Titel Académie universelle des jeux zahlreiche Neuauflagen erlebt (zur Textgeschichte Sanchez, 2014), und der zuerst 1674 anonym erschienene Compleat Gamester, der in späteren Auflagen dem Dichter Charles Cotton (1630-1687) zugeschrieben und heute zumeist so zitiert wird (Seymour, 1734, S. XVIII). Die soziale Bedeutung der Spielbücher mag man daran Ermessen, dass sie auch an die Damen der Gesellschaft adressiert gewesen sind. Die von Seymour besorgten Auflagen des Compleat Gamester schreiben in der Titelei: „Written for the Use of the Young Princesses".

Die Spielbücher erschienen meistens anonym. Man darf sie sich nicht als Originalwerke vorstellen, sondern als Kompilate aus älteren Werken, Exzerpten aus Spezialliteratur, Übersetzungen und zumeist verlorengegangenen Kleinschriften wie z. B. Anleitungen, die die Spielwarenhändler ihren Produkten beilegten. Es ist unwahrscheinlich, dass die Kompilatoren in allen Spielen über eigene Routine verfügten. Und auch an das Publikum muss man eine sehr begrenzte Erwartung haben. Wittmann (1982 und 2011, S. 114-120, 159-161 u. 188-200) schätzt die Zahl der Bücherleser um 1700 in Deutschland auf gerade einmal 100.000 Personen, davon $80 \%$ Akademiker, dazu Angehörige des Adels und des urbanen Bürgertums samt ihrer Frauen. Diese lasen zwar durchaus auch Literatur, die man heute als trivial empfinden würden, aber trotz Schulpflicht in einigen Ländern ist auch die volkssprachliche Buchproduktion im 18. Jahrhundert noch ein sehr elitäres Unternehmen. Erst im letzten Drittel verbreitern sich Lesepublikum, Bücher- und Zeitungsproduktion in erheblichem Maße, wobei der Markt allerdings auch durch abnehmende Wiederholungslektüre befeuert wird. Nur höchstens $20 \%$

\footnotetext{
${ }^{3}$ Für die Bibliographie der Auflagen kann man Schachbibliographien heranziehen
} (Schmid, 1847, van der Linde, 1874, Bd. 2, S. 50-74; 1881) 
der Bevölkerung dürften tatsächlich zur Buchlektüre befähigt gewesen sein. In den Landschulen waren üblicherweise nicht spezifisch ausgebildete, zwar alphabetisierte, aber häufig selbst noch illiterate Lehrer tätig.

\section{Frankreich}

In den frühen Spielbüchern ist Domino noch nicht enthalten. Den bisher ältesten schriftlichen Nachweis fand U. Schädler in einer 1762 erschienen französischen Zeitschrift, wo eine Spielanleitung für ein Spiel mit 21 Steinen (1-6) für zwei bis fünf Personen samt Abrechnung für die Einsätze angegeben ist (N. N., 1762, S. 141-143). 1770 vermeldet eine deutsche Zeitschrift in französischer Sprache ein 28teiliges Spiel in einem Artikel über Moden und Neuigkeiten aus Paris (N. N., 1770, S. 120). Lhôte (1994, S. 462) wies es in zwei 1771 erschienen französischen Wörterbüchern nach. Zum einen verzeichnet der Vocabulaire françois ein Spiel unter dem Lemma „domino“ (N. N. 1771b, S. 256). Zum anderen nahm die jesuitische Enzyklopädie Dictionnaire de Trévoux das Spiel als Nebenbedeutung in das Lemma für marmoriertes Papier auf (N. N., 1771a, S. 418-19).

Die Académie universelle des jeux enthält zum ersten Mal 1786 eine Dominoanleitung (N. N., 1786, S. 181-201). Dieser Text ist auch vergleichsweise ausführlich. So werden taktische Hinweise für verschiedene Anzahlen zu Beginn gezogener Steine und verschiedene Anzahlen an Mitspielern gegeben. Möglicherweise beruht er auf einer 1780 in Amsterdam und Paris verlegten Broschüre, deren einziges Exemplar in der Französischen Nationalbibliothek mir nicht zugänglich war (N. N., 1780). Dass sie im Titel die „Entscheidungen der besten Spieler" verspricht, lässt vermuten, dass Modellpartien enthalten sind.

\section{England}

In England wird der veraltete The Compleat Gamester um die Mitte des 18. Jahrhunderts von einer Textsammlung des Whistlehrers Edmond Hoyle (1672-1769) abgelöst. Seine Abhandlungen erschienen ursprünglich in Einzelheften und werden später zu einem Handbuch zusammengefasst, das dann zahlreiche Auflagen und Bearbeitungen erfährt. Hoyle ist heute Synonym für englische Spielanthologien, vergleichbar mit Duden für deutsche Rechtschreibwörterbücher, weswegen der Hoyle-Sammler David Levy (2013) ihn auch eher für einen Markennamen als für einen Autoren hält. Der erste Hoyle, der Domino enthält, ist die Bearbeitung durch Charles Jones 1803 - offenbar als Addendum, denn erst in späteren Auflagen wird es in den 
Haupttext integriert, wobei der Text identisch bleibt (Jones, 1803, S. IIIIV; Jones, 1814, S. 182-183). Kurz zuvor hatte der Antiquar Joseph Strutt (1801, S. 240) Domino als „eine kindische Mode, die vor einigen Jahren aus Frankreich importiert wurde" bezeichnet. Auch eine Befragung von Zeitzeugen ergab, dass Domino von französischen Kriegsgefangenen nach England gebracht wurde (F. C. H. 1869 , S. 240).

Eine Biographie des englischen Königs Heinrich VIII. behauptet, dieser hätte im Januar $1530450 £$ beim Domino verspielt (Williams, 1971, S. 121). In der Buchhaltung des Hofes steht „Lost by the King to Domyngo at game, $450 £$ " N. N., 1880). Dieser Domyngo ist offensichtlich der Empfänger der Zahlung, aber nicht der Name des Spiels. Ihm sind auch noch häufiger Spielschulden des Königs ausbezahlt worden, z. B. am 3. 11. 1532: „Lost at dice in Calais to Domyngo and Palmer, 100 cr."

\section{Deutschland, Österreich, Schweiz}

Die bisher ältste deutschsprachige Erwähnung, wenn man von der oben genannten deutschen Zeitschrift in französischer Sprache absieht, findet sich 1776 im Programm eines philantropischen Internats in der Schweiz von Salis, 1776, S. 357-358):

[Domino] lassen wir jeden nur eine Zeitlang mit den gewöhnlichen Nummern spielen. Allein so bald er einige Fertigkeit darinnen erlangt hat, muss er auch das historische und geographische Domino spielen lernen. Dergleichen Spiele sind bey uns von verschiedener Art. Statt der Nummern stehen auf den Täfelgen Namen von Städten, Flüssen, Ländern, Helden, Fürsten, welche die Spielenden eben so, wie die Dominotäfelgen, nur nach anderen Regeln, anlegen müssen.

Hinweise auf solche propädeutischen Dominos finden sich mehrfach am Ende des 18. Jahrhunderts (Zimmermann, 1778, S. 1606; Krünitz, 1791, S. 344). Lehrspiele im akademischen Unterricht sind bereits seit dem Hochmittelalter bekannt (Borst, 1986) und Lehrkartenspiele im Besonderen seit dem frühen 16. Jahrhundert, als deren Erfinder der Franziskanermönch Thomas Murner gilt (Hoffmann, 1983, S. 38-43). Der Spielwarenhändler Peter Friedrich Catel (1790, S. 54), der in Berlin einen Nürnberger Laden betrieb, beschreibt in seinem Katalog das geographische Domino so:

Geographische Spiele sind in 49 oder 25 Stücken zerschnittene Homannische und Schreibersche Landkarten, auf Pappe geklebt und in einem pappenen Futteral, nebst einer gedruckten 
Beschreibung dieses Spieles. Es wird ungefähr so, wie das Dominospiel, gespielt und dient ganz vortrefflich dazu, die Jugend mit der wahren Lage der Länder bekannt zu machen.

Daraus wird ziemlich klar, dass hier kein Domino gemeint ist, sondern ein Puzzle, aber dieses Wort wurde erst nach dem zweiten Weltkrieg entlehnt Bekkering, 2005, S. 71). Etwas mehr Ähnlichkeit hat das Regentenspiel des Pädagogen Gutsmuths (1796, S. 353-354):

Man schreibt die Namen der Regenten, ihrer Gemahlinnen und Residenzen einzeln auf Stückchen Kartenblätter oder Pappdeckel, die nicht größer als ein Dominostein sind; vertheilt sie, und lässt Domino damit spielen. Da legt einer den König von England auf; wer die Königin hat legt sie daran, dann folgt der dritte mit der Residenz. Wer die Residenz hat, fährt jedesmal fort, wieder einen neuen König anzulegen. Wer alle drey besitzt, legt gleich alle drey hintereinander hin, u. s. w.

Während die ältesten deutschen Erwähnungen des Domino nahezu genauso alt sind wie die französischen, dauert es erstaunlich lange, bis gedruckte Anleitungen in der Spieleliteratur erscheinen. Das Spielbuch Christian Gottfried Flittners (1770-1828), erstmals 1816 (bzw. 1810 mit anderem Titel und Pseudonym) erschienen, nimmt Domino in der zweiten Auflage 1819 auf, wobei von den Druckplatten auch kleinere Broschüren hergestellt wurden, was die etwas eigenwillige Paginierung des Gesamtwerks erklärt (von Düben, 1819, Kap. XIX, S. 99-104; 1820, S. 99-104). Ebenfalls 1819 erschien das erste Heft des Archivs der Spiele mit einer kurzen Anleitung, in der betont wird, dass es vor allem in Frankreich und Süddeutschland verbreitet ist (N. N., 1819, 122-124). Das langlebigste deutsche Spielbuch, das bereits seit 1705 erscheinende Das neue königliche L'Hombre ..., enthält 1808 noch kein Domino (N. N., 1808). Das ändert sich 1821, wie aus einer Verlagsanzeige hervorgeht (Herold und Wahlstab, 1821). Die Ausgabe von 1846 beschreibt es nur in einem schmalen Text (N. N. 1846b, S. 273-274). Das seit 1795 in Wien erscheinende Neueste Spielbuch nimmt es erstmals in der Auflage von 1829 auf, allerdings klafft zur Vorgängerauflage von 1805 auch eine ungewöhnlich lange Lücke (N. N., 1829, 2. Abschnitt, S. 35-44). Der anonyme Autor zählt es zu den Spielen, die er zuvor schon einmal bearbeitet hat (N. N., 1829, S. V). Sehr wahrscheinlich ist er identisch mit dem Verfasser einer ebenfalls in Wien verlegten selbstständigen Broschüre, deren Vorrede auf Herbst 1826 datiert ist (N. N. 1828). Der Autor schildert, dass er als Kind vor mehr als 
30 Jahren ein Dominospiel mit 21 Steinen ohne Blanke besaß, daran aber ob der geringen Komplexität kein großes Interesse entwickelt hätte. Erst die Einführung von Spielen mit 45 Steinen hätte zu einer Popularisierung geführt, die alle österreichischen Städte beträfe. Domino soll, wie die meisten Spiele, aus Frankreich stammen, wo es schon vor 12 Jahren in Mode gewesen sei. Der Autor erwähnt auch ausdrücklich, dass in Kaffeehäusern üblicherweise um Geld gespielt wurde. Zudem wendet er sich auch explizit an Leserinnen und bespricht mehrere Modellpartien (Abb. 3).

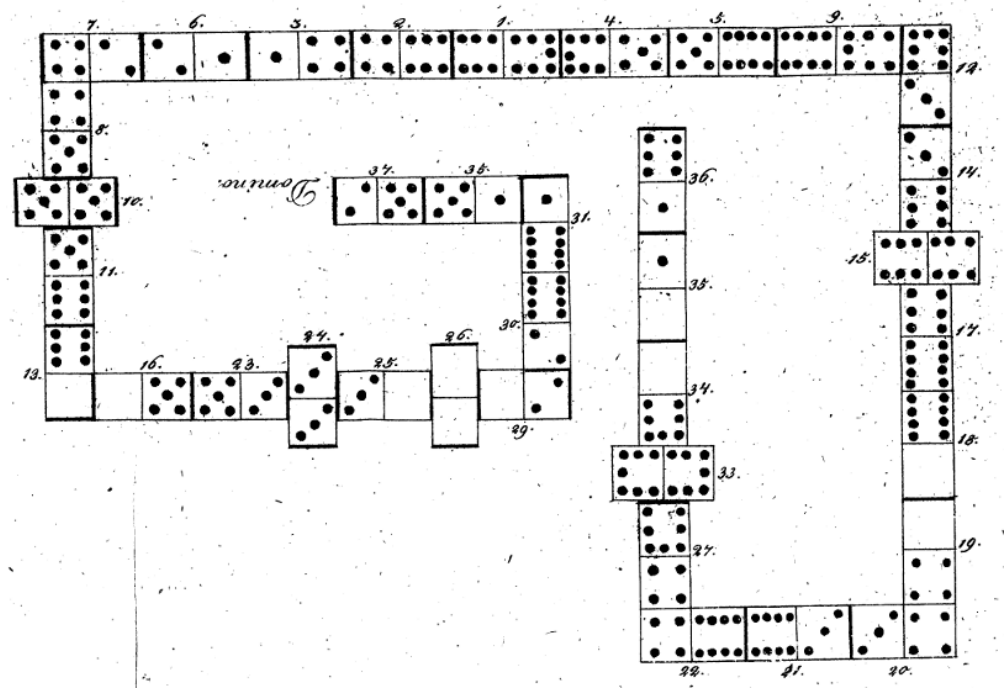

Abbildung 3: Modellpartie aus N. N. (1828). Die Pasche werden quer gelegt, was vermutlich das schnelle Erfassen des Bildes erleichtert. Die Zahlen an den Querrillen geben die Nummer des Spielzuges an. Dazu gehört ein Erläuterungstext.

So ganz sind in Deutschland allerdings französische Verhältnisse nie erreicht worden. Auch noch nach der Franzosenzeit finden sich Reiseberichte von Deutschen, die sich über die Dominobegeisterung unter Erwachsenen in Frankreich wundern (N. N., 1825b; Mohl, 1845, S. 163).

\section{Niederlande und Skandinavien}

In den Niederlanden wurden im 18. Jahrhundert sehr viele französische Bücher gedruckt, weil die Druckkosten billiger waren und sich Probleme mit der französischen Zensur umgehen ließen (Furstner, 1985, S. 39 u. 68-71). Die beiden französischen Dominoanleitungen aus den 1780er Jahren sind in den Niederlanden (mit-)verlegt worden (N. N., 1780, 1786). Die älteste Erwähnung in niederländischer Sprache stammt aus einer Übersetzung des 
philantropischen Internatsprogramms (von Salis, 1777, S. 329). Zudem findet sich Domino in den Erinnerungen eines deutschen Veteranen der Befreiungskriege 1813-1815, der 1814 in s'Hertogenbosch (Noord-Brabant) einquartiert war und schildert, dass es dort in den Gastwirtschaften viel gespielt wurde (Kretzschmer, 1838, S. 171).

In Skandinavien ist bereits 1796 ein Spielbuch in dänischer Sprache erschienen, das mir nicht vorlag. Die zweite Auflage von 1802 enthält eine kurze Dominoanleitung für 21 oder 28 Steine (Jørgensen, 1802, 372-373).

\section{Spanien und Portugal}

Ein frühes Spielbuch in spanischer Sprache habe ich nicht ausfindig machen können. Die älteste Quelle hier ist eine Verkaufsanzeige eines französischen Importeurs in einer Madrider Tageszeitung (Millot, 1802). 1829 veröffentlicht ein Französischlehrer Dialoge, die die Unterschiede zwischen Spanien und Frankreich erklären sollen (Dupuy, 1829, S. 78-81). Demnach haben die Franzosen Domino schon lange, die Spanier erst seit kurzem. Man spiele es kaum zu zweit, zudem gehört es zu den Spielen, die französische Frauen öffentlich und kompetent spielen können - anscheinend auch dies im Gegensatz zu den spanischen. Die älteste Anleitung, die auszumachen war, stammt erst aus einer Heftchenserie von 1839 (P. F. y P. 1839). 1845 spekuliert ein Lexikon über die Herkunft des Spiels „von den Juden, Griechen und Chinesen“ (Rodriguez, 1845, S. 195).

In Portugal hingegen ist schon 1806 ein fünfbändiges Spielbuch mit Domino erschienen (N. N. 1806). Mir war nur der erste Band zugänglich. Er enthält aber ein Gesamtinhaltsverzeichnis, aus dem hervorgeht, dass der letzte Text überhaupt Domino behandelt.

\section{Italien}

Der Ethnologe Culin gibt an, in Brockhaus' Conversations-Lexicon gelesen zu haben, dass Domino Mitte des 18. Jahrhunderts aus Italien über Frankreich in Deutschland eingeführt wurde (Culin, 1895, S. 530). Tatsächlich steht das dort: N. N., 1865, S. 442-443. Der englische Arzt und Spielesammler Robert C. Bell (1979, Bd. 1, S. 162) hat diese vage Information in seinem vielzitierten, zuerst 1960 erschienen Buch nachgerade viral verbreitet. Jedoch enthält selbst Il giuoco pratico, das seit 1753 erscheint, es 1820 immer noch nicht (N. N. 1820). Der älteste italienische Beleg, der auszumachen war, stammt erst von 1825 und ist eine auszugsweise Übersetzung aus der Académie universelle des jeux (N. N., 1825a, S. 207-215). Da sich nur spätere Auflagen 
nachweisen lassen, wurde vermutlich der Titel geändert. Allerdings findet sich das Spiel bereits 1810 in einem deutsch-italienischen Handelswörterbuch (Cunradi, 1810, S. 77 u. 228).

\section{Etymologie}

Zunächst einmal ist festzustellen, dass der ursprüngliche Name des Spiels der Singular domino war. Das Französische (jeu de dominos) und das Englische (Dominoes) benutzen im modernen Sprachgebrauch den Plural und bezeichnen nur den einzelnen Spielstein mit dem Singular, während das Deutsche und Italienische letzteren weiterhin als Spielnamen verwenden. Alle oben genannten französischen und englischen Quellen verwenden ebenfalls den Singular, so dass kein Zweifel über die ursprüngliche Form bestehen kann. Der ältesten Beleg für die Pluralform im Französischen stammt von 1803 (Cambry, 1803, Tab. 59), danach waren offenbar beide Formen in Gebrauch. Der älteste englische Plural findet sich bei Watts (1829, S. 163).

In der Wikipedia (o.J.) wird die Theorie verbreitet, ein schwarzer Priestermantel bzw. ein Ballkleid hätten Pate für den Namen gestanden, oder aber vom Siegesruf eines leidenschaftlich spielenden Abtes „benedicamus domino!", daher auch die Informationen von Auler und Hiller (2015, S. 53). Die ungenannte Quelle dafür dürfte ein früherer Lektor des Verlages von Otto Maier („Ravensburger Spieleverlag") sein, der das aber als spekulativ ausweist (Glonnegger, 1988, S. 78-79; ähnlich: Endrei, 1988, S. 69). Die Manteltheorie findet sich 1910 in der Encyclopcedia Britannica (N. N., 1910, S. 404-405), ist dort aber als Hörensagen gekennzeichnet. Die Ausgabe von 1855 verzeichnet hingegen nur das (Masken-)Ballkleid (N. N., 1855, S. 100). Der von Jesuiten verfasste Dictionnaire de Trévoux lehnte diese Erklärung 1771 implizit ab (N. N., 1771a, S. 418-419). Er unterscheidet semantisch nämlich zwei homonyme Lemmata domino, einmal für den Priestermantel mit der Nebenbedeutung Ballkleid und einmal das oben erwähnte marmorierte Papier mit dem Spiel in der Nebenbedeutung. Auf das Maskenkleid verweist die älteste Quelle von 1762, wenn sie schreibt, der Name stamme aus dem Karneval, bei dem das Spiel häufig ausgeübt wurde (N. N., 1762 , S. 141).

Die Académie universelle des jeux bezeichnet den Gewinn eines Spiels durch Legen des letzten Steines eines Spielers als faire domino und das Neueste Spielbuch folgt ihr darin mit „einen Domino machen" (N. N., 1786, S. 182; N. N., 1829, 2. Abschnitt, S. 38). In den englischen Büchern ist dieser Ausdruck zwar nicht angegeben, aber offenbar rief der Gewinner „Domino!“ 
(Hooper, 1882). Der französische Maler und Karikaturist Honoré Daumier (1808-1879), der Domino mehrfach thematisierte 4 schuf 1839 gar eine Karikatur, in der der Gewinner seinem Gegner „Domino!!" triumphierend ins Gesicht ruft (Abb. 4). Auch im dänischen Spielbuch ist dieser Ausruf angegeben (Jørgensen, 1802, S. 373), und der Wiener Anonymus schreibt es in seinen Mustertafeln, um das Spielende zu markieren (Abb. 3). Einen analogen Ausruf beim Auspielen der letzten Karte faire capot (capot $=$ Haube, Kapuze) gab es im Piquet (Pikett), einem schon im 17. Jahrhundert beschriebenen Kartenspiel (Weekley, 1911; 1921, Sp. 467-468; N. N., 1694, S. 145; N. N. 1718, S. 83). Diese Deutung stützt die Annahme, der Priestermantel hätte Pate gestanden, da er über eine Kapuze verfügte.

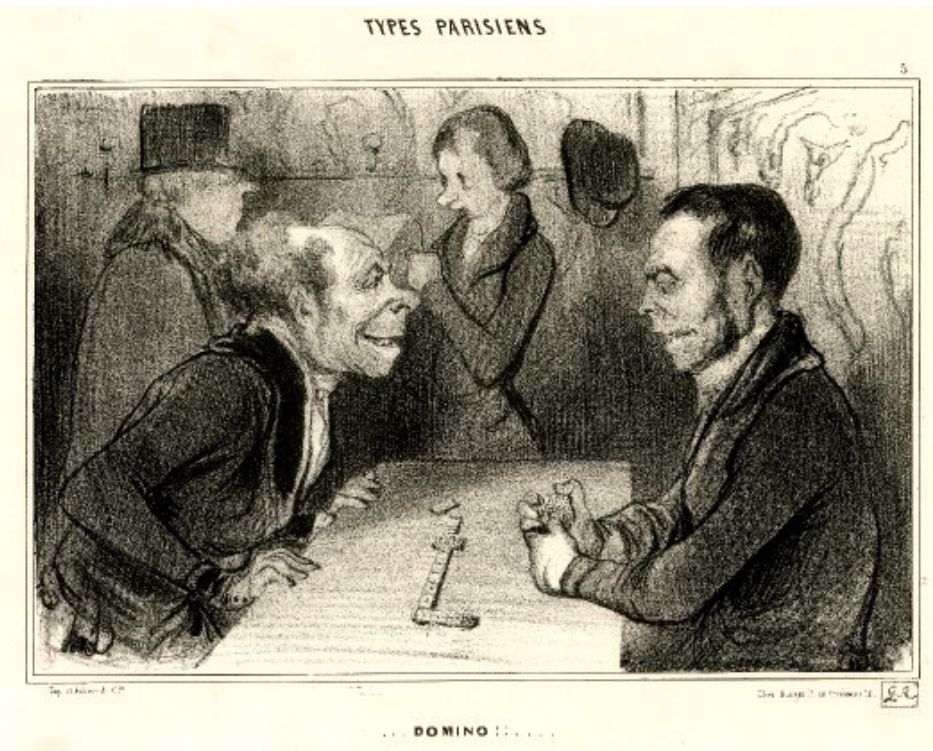

Abbildung 4: „Domino!!“ Karikatur aus der Serie Types Parisiens von Honoré Daumier (18081879). Lithographie, Frankreich, 1839.

Etwas anders nuanciert der Brockhaus 1865: „Wer zuerst sämmtliche Steine abgesetzt, ist ,Domino" (d. i. Herr) und hat das Spiel gewonnen" (N. N.) 1865, S. 442-443). Auch hier wird keine Verbindung zu dem ebenfalls verzeichneten Kleidungsstück gezogen. Die Deutung als Herr wurde dann mit Vorbehalt in den Etymologieduden übernommen (Drosdowski und Grebe, 1963, S. 115). Die ersten Ausgaben des Brockhaus verzeichnen das Spiel leider nicht, aber sie kennen noch das Buntpapier, das in den späteren fehlt

\footnotetext{
${ }^{4}$ Eine von Privatsammlern betriebene Datenbank http://www.daumier-register.org zu Daumier enthielt am 23. 3. 2016 neun Karikaturen mit Dominobezug.
} 
(N. N., 1815, S. 214).

Der Verweis auf das französische Wort für marmoriertes Papier eröffnet aber die Möglichkeit zu einer sehr viel einfacheren Deutung des Namens. Schon 1723 wird es als veraltet bezeichnet, es gab aber dem Gewerbe der dominoterie seinen Namen (Savary des Bruslons und Savary, 1723, Sp. 17161718). Darunter versteht man die Herstellung und den Handel mit bunten Papieren, seien sie bemalt, bedruckt oder chemisch behandelt, marmoriert, ornamental oder figürlich (Thümmler, 1998, S. 49-55). Der Name stammt von den Heiligenbildchen, die zu den wichtigsten Waren dieses Berufsstandes gehörten. Dominopapiere dienten vorwiegend als Auslegeware, um z. B. Möbel, Behältnisse und Bucheinbände auszuschlagen. Zudem benutzte man sie in Frankreich bis an das Ende des 18. Jahrhunderts als Surrogat für die teureren Textiltapeten. Die Spielkartenmacher waren spezialisierte dominotiers (N. N., 1846a, S. 112): „Die Spielkarten sind einer der Zweige der dominoterie" (Grosley, 1813, S. 15). Papierene Dominospiele, wie sie im nächsten Kapitel beschrieben werden, wurden also von dominotiers hergestellt.

Alternative Namen lassen sich kaum nachweisen. Aus Deutschland ist einmal Täfelchen-Spiel belegt (Krünitz und Korth, 1833, S. 122-127). Ein Idiotikon der Schweizer Region Appenzell nennt sowohl das Spiel als auch die einzelnen Steine Brittli (Brettchen) (Tobler, 1837, S. 76).

\section{Spielmaterial}

Ein europäisches Dominospiel besteht in der Grundform aus 28 Steinen, die die Werte 0 bis 6 in allen Kombinationen genau einmal enthalten. Allerdings gibt es Spiele ab 21 Steine und auch welche ohne Null. Bei der Produktion von Spielsteinen aus harten Materialien sparen die Blanken eine ganze Menge Bohrungen, allerdings sind sie beim Spiel um Geld hinderlich. Da bei gesperrten Spielen (keiner kann alle Steine ablegen) die Punkte der bei den Spielern verbliebenen Steine bilanziert werden, muss eine Behandlung für die Doppelnull vereinbart werden (N. N., 1828, S. 49-50). Für Dominosteine gibt es vier wesentliche Quellengattungen:

1. Physische Objekte aus Sammlungen und Ausgrabungen

2. Bescheibungen in den Spielbüchern

3. Preislisten, Musterbücher und Warenkataloge der Händler und Produzenten

4. Bildquellen

Board Game Studies Journal 10, pp. 61-100 DOI $10.1515 /$ bgs-2016-0004 


\section{Objekte, die älter sind als die schriftlichen Quellen}
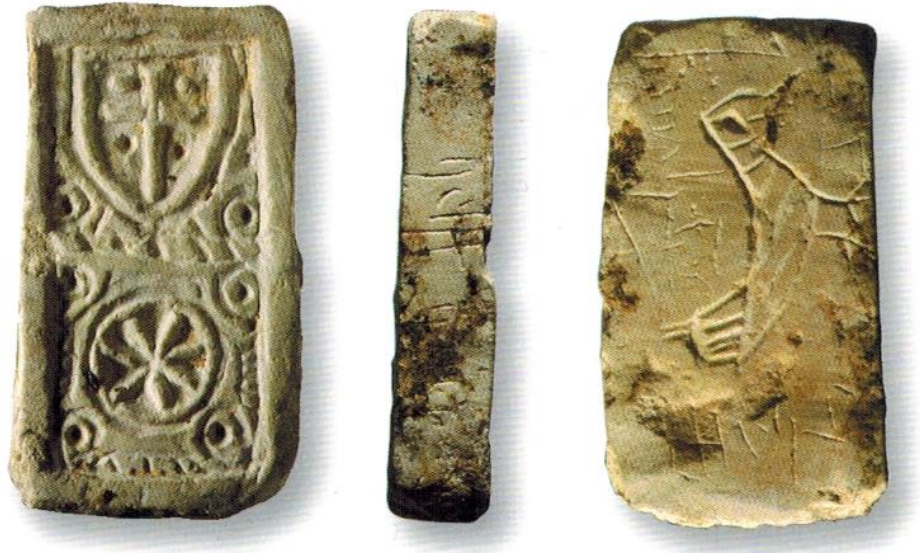

Abbildung 5: Dominostein (2/4) aus Kalkstein aus Einbeck (Lkr. Northeim, Niedersachsen), Altstadtbereich „Petersilienwasser“, Zehnthof, um 1500. Größe 25,5 × 13,5 × $10 \mathrm{~mm}$. Quelle: Heege und Roth-Heege, 2002

Materielle Belege, die älter sind als die schriftlichen Ersterwähnungen, sind ziemlich selten. Der älteste Dominostein in Europa stammt aus Einbeck (Lkr. Northeim, Niedersachsen) aus einem Schichtzusammenhang der Zeit um 1500 und ist aus Kalkstein gefertigt (Abb. 5) (Heege, 1998, Abb. 3; Heege und Roth-Heege, 2002, S. 320-321; zur Fundstelle: Teuber, 2009). Der Stein ist durch sekundäre Ritzungen auf der Rückseite und an den Schmalseiten für wettkampfmäßiges Spiel unbrauchbar gemacht worden und hat deshalb wohl zuletzt als Kinderspielzeug gedient. Bemerkenswert ist allerdings die aufwendige Machart, zumal es sich bei den beiden Symbolen sehr wahrscheinlich um Spielkartenfarben des italienischen Bildes handelt. Das besteht aus Münze, Schwert, Becher und Stab, von denen die ersten beiden hier zu sehen sind. Diese Symbole dienten auf Spielkarten als Zählzeichen, denn der Aufdruck von Zahlen wäre bei einem überwiegend analphabetischen Publikum nicht sehr hilfreich gewesen. Auf den Bildkarten haben sich die Kartenmacher meist bemüht, die Farbzeichen in das Bild zu integrieren. Spielkartenverbote sind in Europa seit 1377 nachgewiesen, dann allerdings in dichter Folge. Spielkarten sind seit dem zweiten Viertel des 15. Jahrhunderts überliefert, wenn auch zunächst nur in sehr geringer Stückzahl. Die regionalen Bilder stabilisieren sich erst im 16. Jahrhundert, bis in dessen Mitte gibt es zahlreiche verschiedene Bilder - auch mit mehr als vier Farben (einen ausgezeichneten Überblick über die Erforschung früher Spielkarten und die Entwicklung der Farbsysteme bietet Hoffmann, 1998, S. 9-119). Italienische 


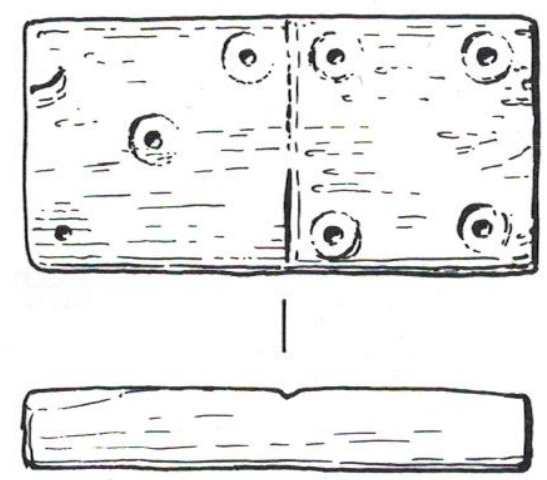

79A0665

Abbildung 6: Beinerner Dominostein (3/4) von der 1545 im Ärmelkanal gesunkenen Mary Rose. Größe 25,8 × 13,3 × $4 \mathrm{~mm}$. In der Originalpublikation ist ein falscher Maßstab angegeben (4 statt korrekt $2 \mathrm{~cm})$.

Farben sind in in der frühen Phase auch nördlich der Alpen nachgewiesen (Schreiber, 1937, S. 11-29 Nr. 7, 10, 15, 16; Hoffmann, 1998, S. 69-82). Es stellt sich natürlich die Frage, wozu Spielkartenfarben auf Dominosteinen dienen sollen? Aus den heute bekannten Regeln gibt es keine Antwort darauf; vielleicht ist es auch eine bloße Verzierung. Möglich wäre eine Art Bilderdomino, wo man statt Nummern Bilder verwendet, so wie sie für Vorschulkinder heutzutage in Gebrauch sind. Immerhin machen diese Symbole bei einem Satz von mindestens 21 Steinen in der Produktion einige Mühe. Es ist keine Ware der untersten Preiskategorie.

Am 19. Juli 1545 sank das englische Kriegsschiff Mary Rose während eines Seegefechts im Solent (der Meerenge im Ärmelkanal zwischen Southampton (Hampshire) und der Isle of Wight). In den 1970er Jahren wurden Unterwassergrabungen durchgeführt, bis sie 1982 gehoben wurde. Auf ihrem Oberdeck wurde bugseitig ein Dominostein aus Knochen gefunden, dessen Augen als Rillen ausgeführt sind (Abb. 6) (Redknap, 2005, S. 135 u. 140141). Der Fundkontext auf statt im Schiff wird als unsicher bezeichnet, dies allerdings, weil man eine so frühe Datierung eines Dominosteins für fragwürdig hält und nicht etwa aus befundimmanenten Gründen.

In Objektdatenbank der Stadtarchäologie von Southampton finden sich mehrere Dominosteine (Southampton City Council, 2014). Zwei aus derselben Grabung (A.2000.84.862 und A.2000.84.915) sollen sogar noch in das Mittelalter datieren (Abb. 7). Da allerdings kein Literaturverweis angege- 
ben ist, ist sie offenbar noch nicht wissenschaftlich ausgewertet worden. Ein Dominostein aus der High Street (A.2000.78.161) gehört wahrscheinlich in das 18. Jahrhundert (Abb. 8) (Platt und Coleman-Smith, 1975, S. 274 und Abb. 249 Nr. 1950). In Plymouth (Devon), ebenfalls an der englischen Südküste, datiert ein stratifizierter Stein (6/0) mit ausgehöhlten Augen (ca. 27 $\times 13 \times 4 \mathrm{~mm}$ ) in das 17. Jahrhundert (Fairclough, 1979, S. 128-129, Nr. 40 - die Zeichnung ist ziemlich schematisch). Aus Grabungen in einem Dominikanerkloster in Oxford (Oxfordshire) wird der Fund eines Belegs des 16. oder frühen 17. Jahrhunderts gemeldet (Abb. 9) (Lambrick und Woods, 1976, S. 218-219, Nr. 50 [M. Henig]; ein ähnlicher Dominostein ist nicht stratifiziert). Ein Engländer will ein Spielset seit der Mitte des 18. Jahrhunderts in Familienbesitz haben, aber es gibt keine Möglichkeit, diese Behauptung zu prüfen (Holme, 1921).

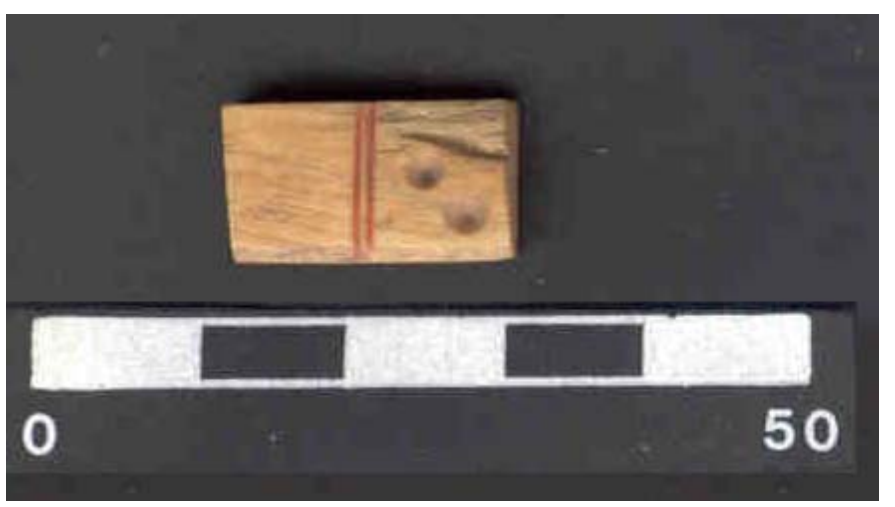

Abbildung 7: Beinerner Dominostein aus Southampton (wohl 0/3) aus Ausgrabung, vorläufig als mittelalterlich datiert.

Ein weiterer früher Beleg zu Beginn des 17. Jahrhunderts ist ein im Amsterdamer Rijksmuseum verwahrtes beinernes Spiel mit Kästchen aus Flandern oder den Niederlanden, das auf jedem Stein eine mehr oder wenige groteske Miniaturbüste und Punktleisten an jeder Schmalseite zeigt (Abb. 10) (Zangs und Holländer, 1994, Nr. E5 [J. Göricke]). Ob bei einem 20teiligen Parallelensemble wirklich die Punktleisten sekundär entfernt wurden, mag hier dahinstehen (Zangs und Holländer, 1994, Nr. E6 [J. Göricke]).

\section{Der Horizont der frühesten schriftlichen Erwähnungen}

Die französische Zeitschrift von 1762 bezeichnet die Steine als cartes und meint damit wohl wirklich Spielkarten. Sie erwähnt nämlich auch elfenbeinerne Ausführungen als lames („Platten“), die nicht so schnell verschmutzen. 


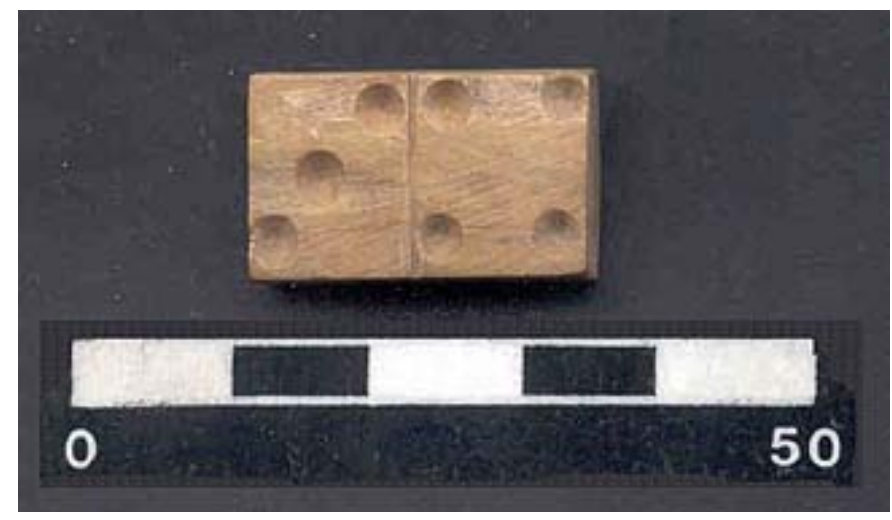

Abbildung 8: Beinerner Dominostein aus Southampton (3/4), wahrscheinlich 18. Jahrhundert. Größe $23 \times 14 \mathrm{~mm}$.

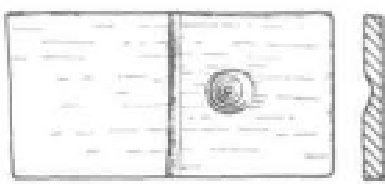

Abbildung 9: Beinerner Dominostein (0/1) aus dem Dominikanerkloster in Oxford. Größe $31 \times$ $15 \times 1,5 \mathrm{~mm}$.

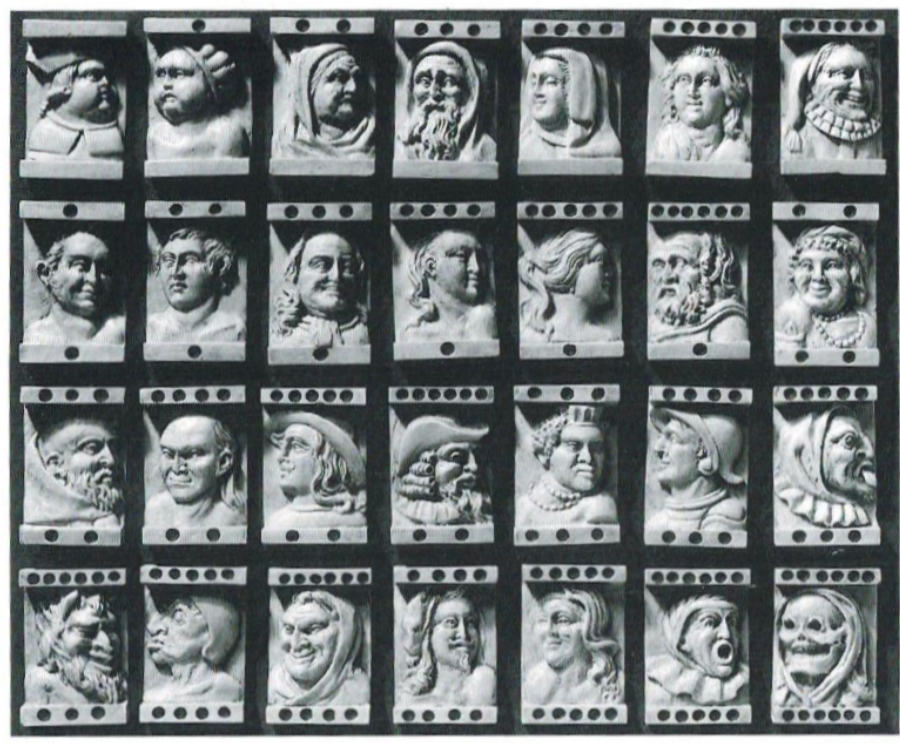

Abbildung 10: Beinernes Reliefdomino aus dem Rijksmuseum Amsterdam, Flandern oder Niederlande, Anfang 17. Jahrhundert. Größe der einzelnen Steine ca. $40 \times 28 \times 9 \mathrm{~mm}$. Quelle: Zangs und Holländer, 1994 , Nr. E5. 
Tatsächlich ist es ein Problem, dass Spielkarten schnell durch Abnutzung individualisiert werden. Im Kartenspiel setzte es sich im Laufe des 16. Jahrhunderts durch, die ursprünglich leeren Rücken mit Mustern zu bedrucken, um die Schmutzwolken zu nivellieren (Hoffmann, 1995, S. 22).

Die Spielbücher beschreiben allesamt ausschließlich Spielsteine aus Hartgeweben; und auch die Zeitschrift von 1770 spricht von tablettes in einem Holzkästchen. In der Académie universelle des jeux werden sie als Würfel (dé) bezeichnet (N. N., 1786, S. 181-201; N. N., 1771a, S. 418-419). Laut Dictionnaire de Trévoux sind die Steine von 1 bis 9 nummeriert. Die Académie universelle des jeux beschreibt den europäischen Normspielsatz mit 28 Steinen von 0 bis 6. Diese sind gewöhnlich aus Knochen oder Elfenbein. Bei besseren und üblicheren Spielsätzen besteht nur die Punktseite aus diesen Materialien, während die Rückseite aus geschwärztem Holz („bois noirci“) gefertigt ist (N. N., 1786, S. 181-182. Die etwas verwirrende Angabe, dass jeder Wert achtfach vorkommt, kommt daher, dass der Pasch jeweils doppelt zählt).

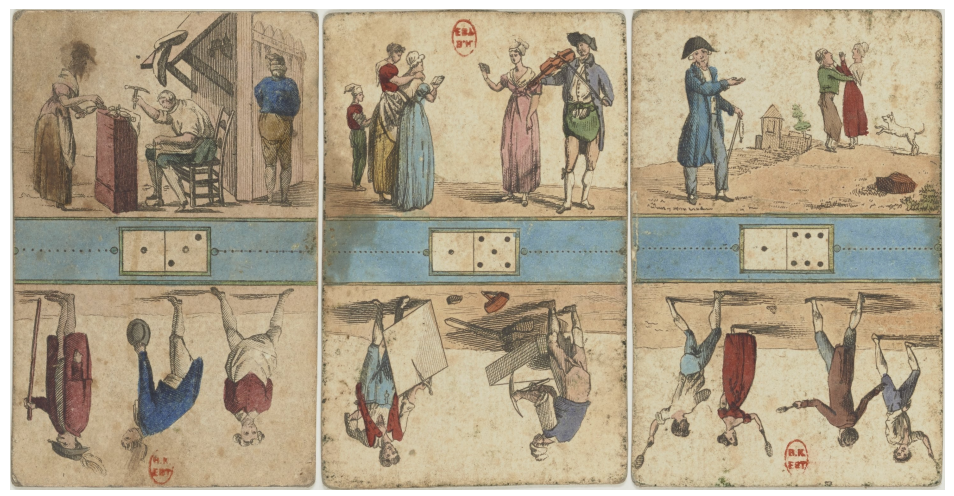

Abbildung 11: Drei Karten eines gedruckten Dominospiels aus der Französischen Nationalbibiliothek (Signatur ark:/12148/btv1b105091818), 1814-1830, Größe der einzelnen Karten 9,1 × 5,9 $\mathrm{cm}$.

In der Französischen Nationalbibliothek haben sich Karten zweier gedruckter Dominospiele erhalten ${ }^{5}$ Ein Spiel ist mit 27 von 28 Karten fast

\footnotetext{
${ }^{5}$ Die Objekte aus der Bibliothèque nationale de France können in deren digitaler Bibliothek Gallica auf http://gallica.bnf.fr/ eingesehen werden. Zwei weitere gedruckte Spiele befinden sich im British Museum, dessen Sammlung auf http:// ww. britishmuseum.org/research/collection_online/search.aspx durchsucht werden kann. Ein Spiel aus Paris von 1800-1875 (Inv.-Nr. 1982,U.4595.1-28) (Willshire, 1876. Nr. F. 102) und ein Bogen eines Spiels mit 21 Steinen, der 1896 mit Teilen der Spielkartensammlung von Lady Charlotte Schreiber erworben wurde (1896,0501.1388). Bei diesem Bogen sind die Augen als Gesichter ausgearbeitet. Leider sind Herkunft und Datierung
} 


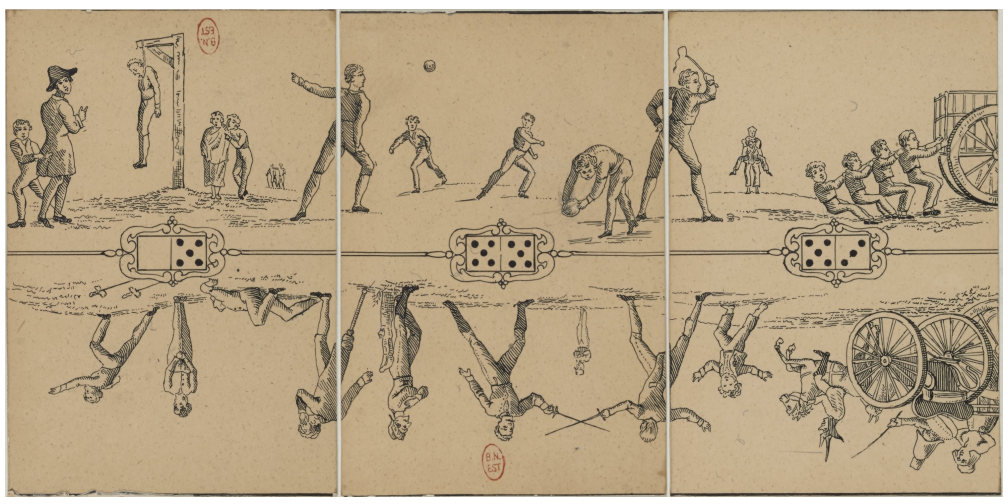

Abbildung 12: Drei Karten eines Myrioramadominospiels aus der Französischen Nationalbibiliothek (Signatur ark:/12148/btv1b10523845m), 1800-1815, Größe der einzelnen Karten 9,6 × 6,7 $\mathrm{cm}$.

vollständig (lediglich der Zweierpasch fehlt) und wird im Katalog auf 18141830 datiert (Abb. 11). Es zeigt auf jeder Karte zwei Szenen, zumeist Berufe oder private Szenen. Ein auf 1800-1815 datierte Spiel, von dem zwölf Karten vorhanden sind, ist noch etwas trickreicher, denn die Schnittkanten setzen sich im Spielverlauf zu zwei immer neuen Bildfriesen zusammen, weil gleiche Augen zwar immer passende, aber jeweils verschiedene Zusammensetzungen produzieren (Abb. 12). Damit es ist gleichzeitig ein Myriorama (von alt-

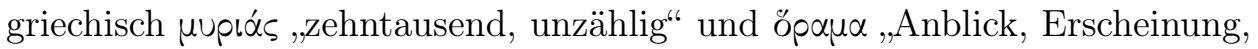
Vision"), also ein zerlegtes Panorama, das sich auf beliebige Weise wieder zusammensetzen lässt. Myrioramen sollen 1802 in Paris erfunden worden sein Oettermann, 1997, S. 67-68). Dieses Spiel ist also ein sehr frühes, aber auch sehr einfaches Beispiel, vor allem, weil es auf gleiche Augenzahl beschränkt ist. Die Funktion als Domino dürfte deshalb im Vordergrund gestanden haben. Von dem Satz gibt es auch eine kolorierte Fassung (van Delft und Botermans, 2004, S. 64-65). Da es sich aber um exakt dasselbe Ensemble ohne Alterungsspuren handelt, wurde hier wohl eine moderne Kopie bearbeitet.

In Nordfrankreich ist in der Stadt Mèru (Dep. Oise, Region Nord-Pasde-Calais-Picardie) seit dem frühen 19. Jahrhundert eine regelrechte Dominoindustrie belegt (Vegas, 1815, S. 262). Ein württembergischer Forschungsreisender, der zwischen 1836 und 1841 in Frankreich war, beschreibt, dass acht Familien mit insgesamt 100 Beschäftigten ausschließlich Dominospiele herstellen (Mohl, 1845, S. 163). Er schätzt den Jahresausstoß an einfachen Spielen nur aus Knochen auf ca. 25.000. Dazu kommen dann noch wertvol-

unklar. 
le Spiele aus Elfenbein und Perlmutt. Besonders kostbare Spiele haben mit Gold eingelegte Augen und werden in Kästchen aus Zitronenholz verkauft. Die wertvollsten Spiele stellen D. und P. Jumel im Nachbardorf Déluge her (auch Jumelle (N. N., 1841, S. 1209 u. 1676)). Während französische Spiele halbkugelförmig ausgerundete Augen haben, werden auch englische Spiele mit konvexen Erhöhungen in der Augenhöhle (soll wohl heißen, als Rillen) hergestellt und sogar mit einem gefälschten englischen Stempel versehen. Mèru hat Dominosteine auch sein Wappen aufgenommen $\sqrt{6}$

Strutt beschreibt ebenfalls ein 28teiliges Spiel von 0 bis 6 aus Elfenbein oder Knochen, bei denen die Rückseiten manchmal geschwärzt sind und die Schauseiten durch einen Strich in der Mitte geteilt werden (Strutt, 1801, S. 240). Der Jones-Hoyle liefert eine gleiche Beschreibung ohne die Schwärzung der Rückseite und die Fertigung aus Knochen (Jones, 1803, S. III). Er erwähnt aber ein verdoppeltes Spiel mit 12 als höchster Punktzahl. Sowohl Strutt als auch der Jones-Hoyle bezeichnen die Dominosteine als „cards“. Etwas später ist auch „stones" belegt (N. N., 1910, S. 404). Das ist recht ungewöhnlich, da das deutsche Spielstein eigentlich mit piece, token oder im Falle von Schachfiguren auch mit man zu übersetzen ist.

Daneben gibt es in England archäologische Funde und museale Stücke aus Gefangenenlagern der napoleonischen Kriege. Das Lager Norman Cross in der Nähe von Peterborough (Cambridgeshire) beherbergte seit 1797 bis 1814 französische, zumeist niedrigrangige Kriegsgefangene, die zunächst aus Marineoperationen stammten. Dort wurden 2009 einige Ausgrabungen vorgenommen (Mytum und Hall, 2013; Wessex Archaeology, 2010a,b). In Norman Cross gab es einen Marktplatz, auf dem die Häftlinge sowohl kaufen als auch verkaufen konnten. Die handwerklich Befähigten stellten dafür insbesondere Knochenschnitzereien her. Unter diesen befanden sich neben allerlei Haushaltswaren auch Würfel und Dominospiele (Abb. 13), die sowohl innerhalb des Lagers als auch nach außerhalb gehandelt wurden. Das Spiel um Einsätze (Kleidung, Essensrationen) konnte für die ohnehin prekäre Existenz der Gefangenen eine nachgerade existenzielle Bedrohung sein.

Beinerne Dominospiele mit der Zuschreibung zu französischen Kriegsgefangenen in England finden sich in mehreren Museen und auch regelmäßig im englischen Antiquitätenhandel. So auch im Museum des schottischen Perth, wo seit 1812 ein großes Kriegsgefangenenlager betrieben wurde (Perth Museum and Art Gallery, o.J.). Leider ist meistens nicht angegeben, wieviel Stei-

\footnotetext{
${ }^{6}$ Auch das polnische Dorf Dominowo (Powiat Średzki, Woiwodschaft Großpolen) hat einen Dominostein im Wappen, aber dabei handelt es sich um ein „redendes Wappen“, also eine bloße Klangassoziation (Heraldik-Wiki, 2015).
} 

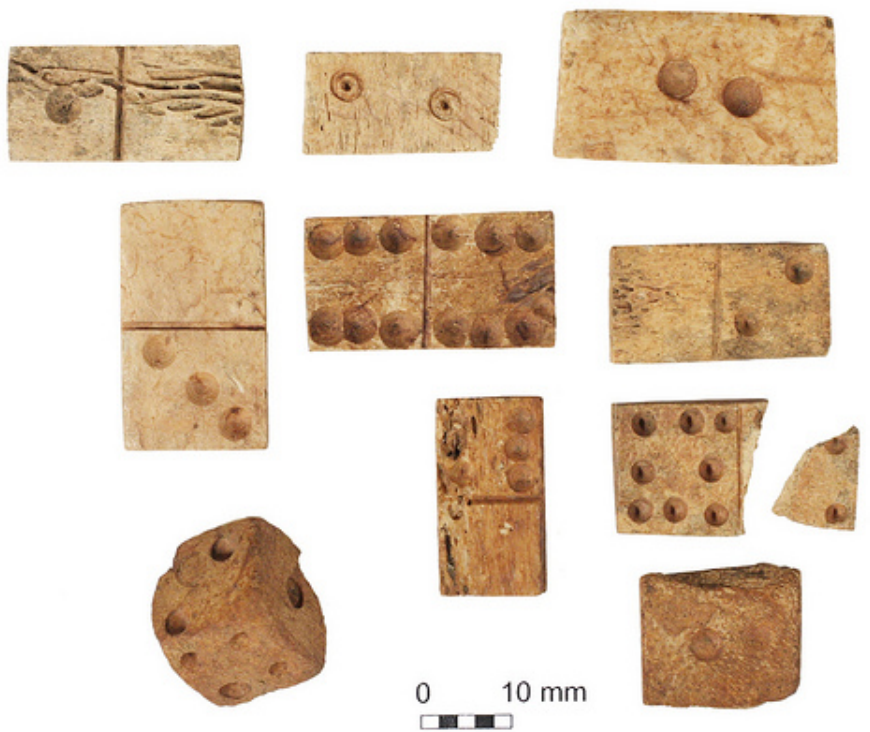

Abbildung 13: Dominosteine und Würfel aus Knochen aus dem Kriegsgefangenenlager Norman Cross bei Peterborough (Cambridgeshire), England, 1797-1814. Quelle: Wessex Archaeology $2010 \mathrm{a}$

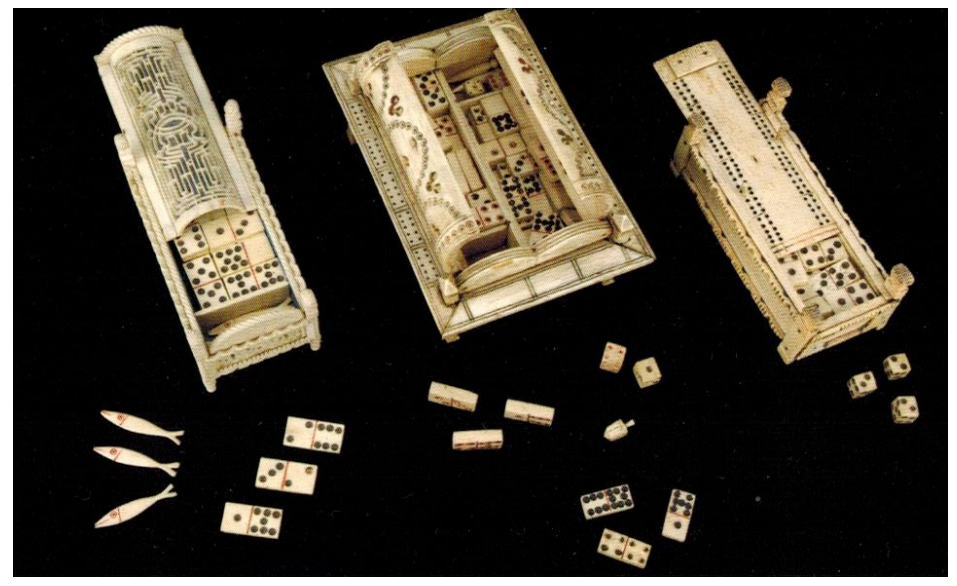

Abbildung 14: Typische French prisoner of war Domino sets aus Bein im Schweizerischen Spielmuseum mit Würfeln und Cribbagemarken. Knochen, Anfang 19. Jahrhundert, keine Maßangaben. Entsprechende Stücke aus dem Antiquitätenhandel sind zwischen 5,7 und $24 \mathrm{~cm}$ lang, meistens zwischen 12 und $16 \mathrm{~cm}$. Quelle: Lhôte 2007, S. 117. 
ne die Sets enthalten. Jedenfalls gibt es Spiele mit bis zu 9 Punkten. Häufig haben die Kassetten oben auch ein Cribbagebrett. Cribbage ist ein Additionsspiel wie 17 und 4 (Black Jack), das schon in The Compleat Gamester beschrieben wird (N. N., 1680, S. 75-79: „Cribbidge“). Da man während des Spiels für bestimmte Kartenfolgen Zusatzpunkte sammeln kann, die sich bei der Schlusszählung schlecht zuordnen lassen, benutzt man Zählbretter mit je 60 Punkten in zwei Reihen, um die Zusatzpunkte zu markieren. Abgebildet sind drei Spielsets, die das schweizerische Spielemuseum in La Tourde-Peilz (Kanton Waadt) erworben hat (Abb. 14) (Lhôte, 2007). Sie zeigen die typische, an ein Bett gemahnende Form mit dem Cribbagebrett an den Langseiten. Ein ebensolches Stück, das inschriftlich als französischer Herkunft ausgewiesen ist und 1798 in England als Hochzeitsgeschenk diente, hat das Museum in Buxton (Derbyshire) angekauft (Buxton Museum and Art Gallery, o.J.). Es gibt aber auch ganz andere Ausführungen, z. B. als doppelkonische Büchse (Bell, 1979, Bd. 1, Tf. XIX). Das Lancaster City Museum (Lancashire) besitzt eine als child's games compendium angesprochene Kassette, in der sich neben einem Dominospiel (0-9) auch Spielkarten aus Knochen befinden (White, 2001). Eine Internetrecherche nach French Prisoner-of-War Dominoes am 6. 10. 2015 erbrachte 39 Angebote im angelsächsischen Antiquitätenhandel. Bei bereits verkauften Losen konnten besonders fein gearbeitete Stücke Preise um ca. $2000 €$ erzielen.

In Deutschland druckt der Schriftsteller Friedrich Nicolai (1733-1811) eine Knochenschnitzerpreisliste aus „Geißlingen" (Geislingen an der Steige, Lkr. Göppingen, Baden-Württemberg) ab, in der Dominospiele mit 36 Steinen zu 10 bis 18 Kreuzern und solche mit 45 Steinen zu 15 bis 24 Kreuzern angeboten werden (Nicolai, 1795, S. 103). Zudem kann man sie im Dutzend zu 1 bis 2 Gulden kaufen, was wohl die Normalausführung mit 28 Steinen meint, weil sich ansonsten für die größeren Spiele Rabatte von mindestens $50 \%$ auf das einzelne Set ergäben. Dieses Angebot zeigt auch, dass sich die Preisliste an Händler richtete und die Endkundenpreise höher gelegen haben müssen. Die Reise fand 1781 statt, der Druck 1795.

Der international renommierte Nürnberger Galanterie-, Spielwaren- und Möbelhändler Georg Hieronimus Bestelmeier (1764-1829), dessen seit 1793 erschienener Katalog als einer der ältesten illustrierten Versandkataloge der Welt gilt, verkauft 1803 nur papierne Spiele: ein „Karten-Dominospiel mit Futteral 10 kr.", bei dem die Punkte als Herzen dargestellt sind (Abb. 15) (Bestelmeier, 1979, Nr. 954). Außerdem ein „Neues Farben-Dominospiel mit 37 illum. Blättern, neue Aufl. 30 kr.", das leider nicht abgebildet ist (Bestelmeier, 1979, Register S. 10, rechte Spalte). Die Werte dürften von 0 bis 7 oder 1 bis 8 gehen, was 36 Stück ergibt (Tab. 1), plus eine Anleitung. Mit 10 


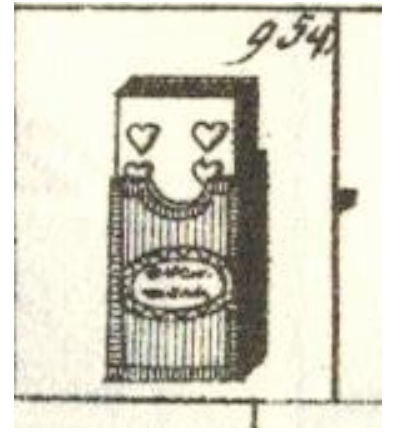

Abbildung 15: Dominospiel in Spielkartenform aus dem Katalog des Nürnberger Spielwarenhändlers Georg Hieronimus Bestelmeier 1803.

und 30 Kreuzern gehören sie zu den billigsten Angeboten. Bemerkenswert ist, dass keinerlei Erläuterungen zum Ablauf angegeben sind. Bestelmeier muss davon ausgegangen sein, dass seine wohlhabende Kundschaft das Spiel regelmäßig kennt. Das von ihm ebenfalls angebotene „neue Wahrsagespiel“ hat sich im Nürnberger Spielzeugmuseum erhalten (Stauss, 2015, S. 366-367). Da ein Teil der Papierkarten zwei Würfelseiten aufgedruckt hat, enthält es praktisch zwei 21teilige Dominospiele.

Tabelle 2: Preise für Dominospiele aus Nürnberg 1838 in Gulden-Kreuzer-Währung nach dem 24-Gulden-Fuß.

\begin{tabular}{|l|c|c|c|c|c|}
\hline Domino-Spiele von Bein, das Stück mit Stein & 21. & 28. & 36. & 45. & 55. \\
\hline ordinäre & 12. & 15. & 20. & 25. & 38. \\
schwarz gebeizt Bein & 24. & 32. & 44 & 58. & 1.10. \\
polirte mit ord. Augen & 21. & 31. & 42. & 50 & $1 .-$ \\
polirte mit eingesetzten Augen & 42. & 52. & 58. & 1.10. & 1.18. \\
pariser Art und gebeiztem Holz & 42. & 52. & 1.4. & 1.20. & 1.40. \\
pariser Art und mit Stiften & 50. & 1.8. & 1.24. & 1.48. & 2.12. \\
mit gebeiztem Holz ohne Stifte & 40. & 48. & 58. & 1.10 & 1.24. \\
mit gebeiztem Holz und geniethet & 42. & 52. & 1.4. & 1.20 & 1.36. \\
pariser Art Ebenholz & 52. & 1.8. & 1.24 & 1.48. & 2.12 \\
pariser Art Ebenholz und geniethet & 1.12. & 1.24. & - & 2.36. & 3.18 \\
mit Ebenholz belegt und geniethet & 52. & 1.4. & 1.16 & 1.28 & 1.50 \\
mit Ebenholz & 42. & 50. & $1 .-$ & 1.16. & 1.40 \\
von schwarz gebeiztem Holz 15 L. lang 10 L. breit & 15. & 18. & 24. & 30. & 36. \\
\hline
\end{tabular}

Eine um 1825 datierende Preisliste eines Münchner Buchhändlers bietet ein „Dominospiel in Figuren dargestellt. 28 colorirte Blätter im Schuber. 20 gr. "in Taler-Groschen-Pfennig-Währung feil (Lindauer, o.J.). Ebenso hat die Spielkartenfabrik von Maximilian Uffenheimer in Guntramsdorf (Niederösterreich) 1828 Dominokarten im Angebot (von Keeß und Blumenbach, 1829. 
S. 640-641). Leider wird die Größe des Satzes nicht genannt. Eine Nürnberger Preisliste von 1838 enthält beinerne und hölzerne Dominosteine in vielen verschiedenen Ausführungen und Nummerierungen (Tab. 2) (N. N., 1838, S. 90). Da angemerkt wird „Bei den Pariser Dominos sind die Steine 20 Linien lang $8 \frac{1}{2}$ L. breit." (wobei auch diese einheimischer Produktion waren), haben französische und deutsche Dominosteine anscheinend tendenziell unterschiedliche Proportionen. Da bei einer Ausführung Maße von 15 L. × 10 L. angegeben werden, sind dies offenbar auch nicht die gewöhnlichen. Es wird auch ein Spiel „mit komischen Figuren in Pappkästchen“ zu 22 Kreuzern angeboten, was offenbar vergleichbar zu dem alten Amsterdamer Spielsatz ist, auch wenn die Ausführung angesichts des relativ niedrigen Preises eher einfach sein muss. Das Neueste Spielbuch beschreibt zwar die Steine nicht näher, gibt aber an, dass ein 45teiliges Spiel (0-8) für bis zu sechs Spieler die seinerzeit gebräuchlichste Variante gewesen sei (N. N., 1829, 2. Abschnitt, S. 35-44). Auch Krünitz und Korth (1833, S. 122-127) nennen Spiele mit 36 oder 45 Steinen, allerdings beziehen sie sich ausdrücklich auf das Neueste Spielbuch.

Das dänische Spielbuch erwähnt Dominosteine aus Stein (Jørgensen, 1802 S. 372). Tatsächlich lassen sich manche weiche Gesteine gut schneiden und waren deshalb auch als Rohmaterial für Schnitzerei in Gebrauch. Ein deutsches Gewerbehandbuch erwähnt Dominosteinproduktion aus Alabaster (Krünitz und Korth, 1833, S. 122). In Zöblitz (heute zu Marienberg, Erzgebirgskreis, Sachsen) wird mindestens seit dem 16. Jahrhundert Serpentin verarbeitet; eine Preisliste aus dem 19. Jahrhundert nennt „Domino-, Würfel- und Schachspiele" (Kluge, 1860, S. 459). In Sawtry (Cambridgeshire, England) wurde jüngst ein in das 18. oder 19. Jahrhundert datierter Stein aus Schiefer gefunden (Miller, 2015). In einer englischen Datenbank für ehrenamtliche Bodendenkmalpfleger (https://finds.org.uk/) finden sich auch mehrere Dominosteine aus Bleilegierungen, die von Sondengängern aufgelesen wurden und über deren Datierung kaum etwas zu sagen ist.

\section{Bildliche Quellen}

Eine frühe Abbildung stammt von dem Augsburger Kupferstecher Johann Jacob Haid (1704-1767) (Dubosc, 1927, S. 56 [ohne Abb.]). Um 1760 entstand ein Schabkunstblatt, das zwei vornehme, adulte Personen verschiedenen Geschlechts beim Dominospielen zeigt (Abb. 16). Leider lassen sich praktisch keine Details des Spiels erkennen. Es beginnt wohl gerade erst, denn es sind nur ausgeteilte, aber keine abgelegten Steine zu erkennen. Dass die Bildunterschrift auf Französisch gehalten ist, kommt auf Haid-Stichen 


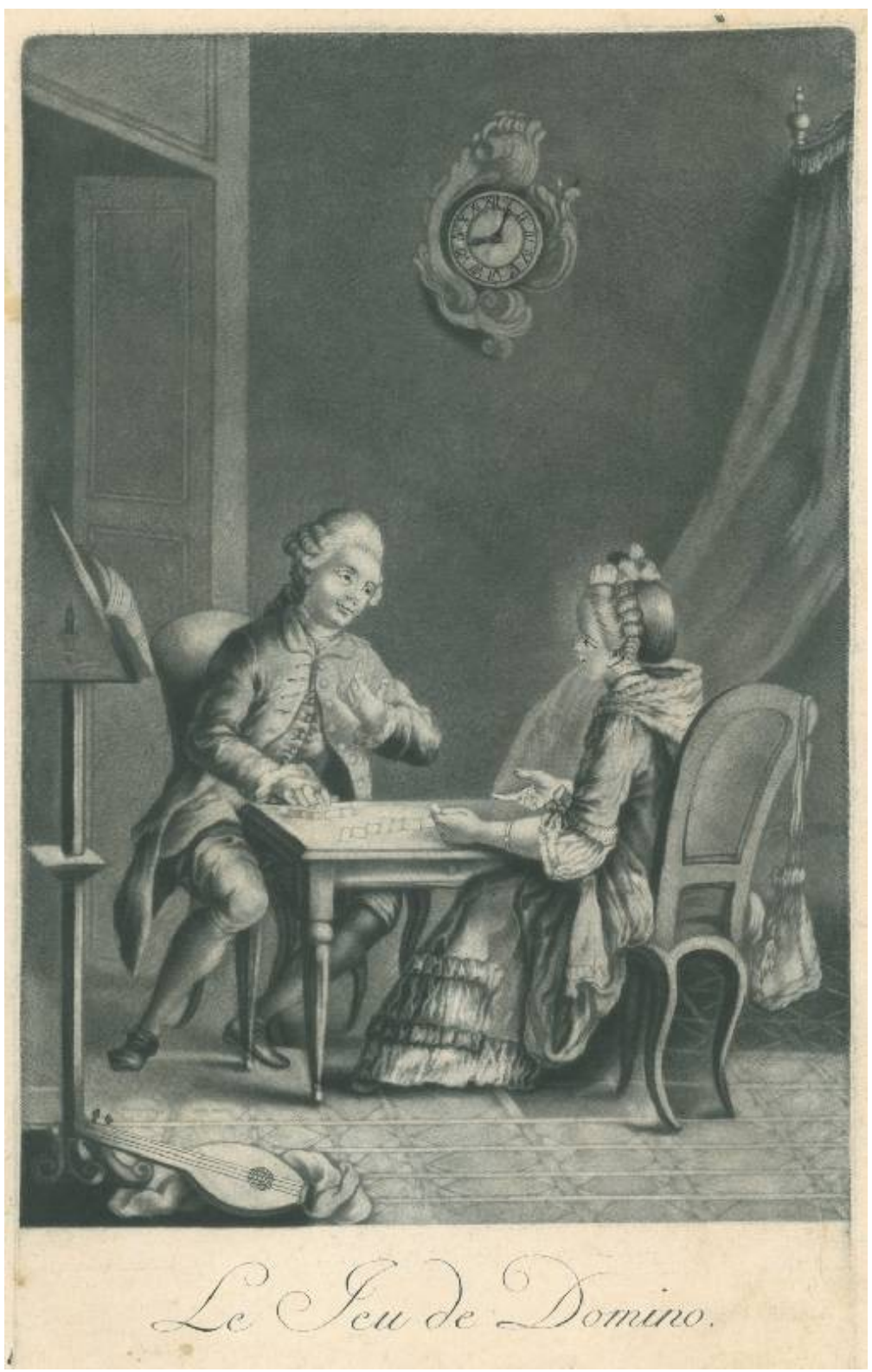

Abbildung 16: „Le Jeu de Domino“. Mezzotintoblatt von Johann Jacob Haid, Augsburg (Bayern, Deutschland) um 1760, 26,5 × 18,5 cm. Bildquelle: Antiquariat P. Bierl, Eurasburg. 


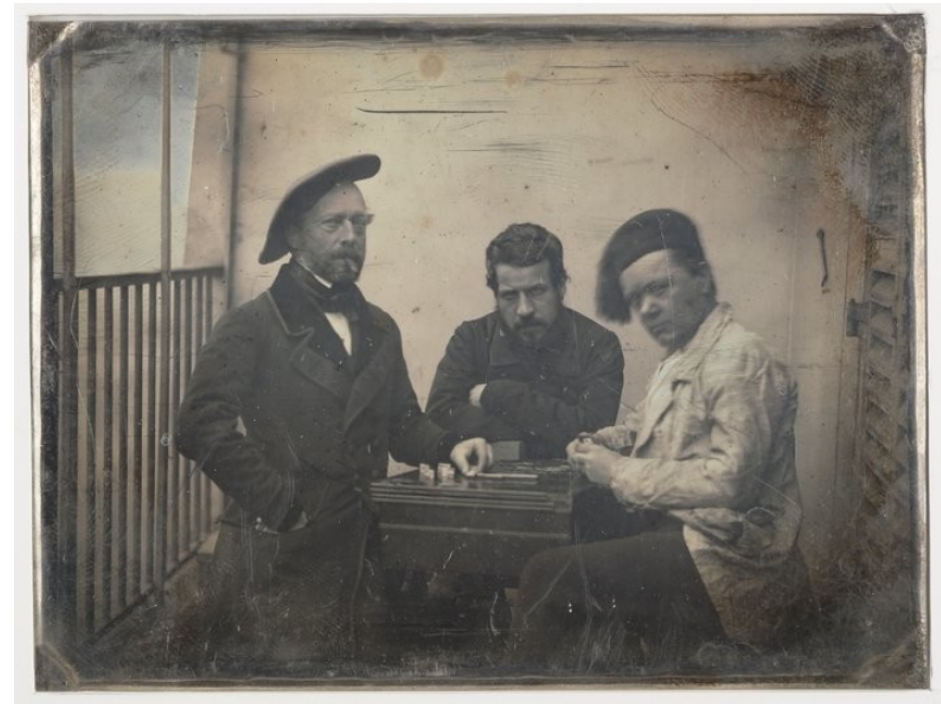

Abbildung 17: Eine Daguerrotypie zeigt von links nach rechts einen unbekannten Mann, den Bildhauer Auguste Clésinger (1814-1883) und den Fotografen Auguste Mestral (18121884) beim Dominospiel. Ca. 1847, $8 \times 10,6 \mathrm{~cm}$, Französische Nationalbibliothek (Signatur ark:/12148/btv1b55009192s).

häufiger vor. Das Blatt hat sicherlich eine amouröse Konnotation. Neben dem Musizieren (Notenständer und Laute) schafft das Spiel eine Situation, bei der sich die Geschlechter treffen können. Auch sehen sich beide Figuren gegenseitig an, anstatt nachdenklich auf die Steine zu blicken, wobei der Mann einen recht verklärten Eindruck macht. Die Uhr legt nahe, dass man sich bereits in den Abendstunden befindet. Dass rechts ein Alkoven in den Bildausschnitt hinein ragt, ist ein ziemlich unmissverständlicher Hinweis auf sexuelle Wünsche. Der Titel ist dann ironisch zu verstehen.

Eine verhältnismäßig alte Abbildung sind Dominosteine in einer Bilderfibel für Leseanfänger von 1815 auf einer Tafel mit Gegenständen, die mit dem Buchstaben D beginnen (Hesse, 1815, S. 31-32). Bei diesen Steinen sind die Punkte durch zwei beinerne Intarsien in einen Holzblock eingelassen.

Ein Ausweis für die Wertschätzung, die Domino in Frankreich erfuhr, sind die oben erwähnten Karikaturen von Daumier und vielleicht auch eine frühe französische Fotografie, die eine Gruppe Künstler beim Dominospiel um 1847 zeigt (Abb. 17). Solche Daguerrotypien waren wegen des verwendeten Filmmaterials (versilberte Kupferplatten) und ohne die Möglichkeit, Abzüge herzustellen, kostspielige Unikate. Die wenigen Gemälde, die nachzuweisen waren, stammen aus der zweiten Jahrhunderthälfte und geben für das Thema 
der frühesten Datierungen nichts her 7

\section{In den Kolonien}

Archäologische Funde zeigen, dass Domino in der Mitte des 19. Jahrhunderts auf der ganzen Welt verbreitet ist und dabei auch mit einer bachtlichen sozialen Diversität imponiert. In den USA ist aber zu beachten, dass ostasiatische Immigranten es direkt aus ihren Heimatländern mitbrachten (Culin, 1895, S. 491). In dem zwischen 1842 und 1849 betriebenen Fort Atkinson in Iowa fand man einen Dominostein in einem Bereich, in dem sowohl Soldaten als auch Kinder von Offizieren lebten (University of Iowa Office of the State Archaeologist, o.J.). In Kenmore, einem Haus, das George Washingtons Schwester kurz vor dem Bürgerkrieg (1861-1865) in Fredericksburg, Virginia erbaute, wurden drei Miniaturdominosteine gefunden (The George Washington Foundation, o.J.). Leider sind sie wegen tierischer Verschleppung nicht genauer datierbar. Das Museum ordnet sie wegen der Miniaturisierung Soldaten aus dem Bürgerkrieg zu. Bei Grabungen in einem historischen Wohnquartier von San Francisco, Kalifornien wurden in fünf Wohnhäusern von Familien mit westeuropäisch klingenden Namen Dominosteine der 1870/80er Jahre gefunden (Gibson, 2009). Auch bei Ausgrabungen in Saloons in der Bergbausiedlung Virginia City (Nevada), die in den 1860er bis 1880er Jahren betrieben wurden, ließ sich Domino neben dem erwartbaren Würfel- und Kartenspiel nachweisen (Dixon, 2005, S. 122-123).

Auf der anderen Seite des Kompasses stammt ein um 1880 datierter Spielstein aus dem ärmlichen Arbeiterviertel Little Lon in Melbourne (Australien) (Museum Victoria, o.J.). In einem Heim für junge Immigrantinnen ohne Familie, das in Sydney von 1848 bis 1886 betrieben wurde, konnte Domino ebenfalls nachgewiesen werden (Davies et al., 2013, S. 90-92).

\section{Die theoretische Summe}

Domino ist in China deutlich älter als in Europa, und es gab dort Varianten, die dem europäischen Spiel stark ähneln. Man kann sich leicht vorstellen, dass

\footnotetext{
${ }^{7}$ Neben den beiden oben genannten von Anker und Simpson handelt es sich um ein Gemälde des englischen Spätimpressionisten Frank Bramley von 1886, das zwei Näherinnen beim Domino zeigt, in der Crawford Art Gallery, Cork (Grafschaft Cork, Irland) (Crawford Art Gallery, Cork, 2014). Ferner eines des Österreichers Hermann von Kern in Privatbesitz, das zwei bürgerliche alte Männer spielend abbildet (Braun, o.J.). Desweiteren ein Gemälde im Besitz des Royal Hospital Chelsea, einem seit 1691 betriebenen Altersheim für Veteranen der britischen Armee in London, das einen Bewohner in der scharlachroten Uniform des Hauses mit Pfeife beim Dominospielen zeigt (Art UK, o.J.).
} 
es sich mit den Spielkarten gen Westen verbreitete. Allerdings gibt es bisher keine historische Verbindung von den europäischen zu den chinesischen Spielkarten, die sich bereits von den indischen fundamental unterscheiden sollen (Hoffmann, 1995, S. 35). Zudem sind die ältesten Nachweise für Spielkarten bei den Arabern jünger als in Europa, auch wenn die Forschung diese Lücke mittlerweile verkleinern konnte (Hoffmann, 1998, S. 19-30). Vor der schriftlichen Ersterwähnung von 1762 sind aus Europa nur sehr verstreute Belege bekannt, die immerhin bis an das Ende des Mittelalters zurückreichen. Mit dem Einbecker Spielstein besteht eigentlich kein Grund mehr, an dem Stein von der Mary Rose zu zweifeln, jedenfalls insoweit dies nur chronologische Gründe hat. Diese frühen Belege schließen auch eine Herkunft des Spiels über den Seeweg aus China ziemlich sicher aus (der Portugiese Vasca da Gama umsegelte Afrika erstmals 1498). In Europa hat Domino weder das analytische Interesse geweckt, das dem Schach und dem akademischen Zahlenkampfspiel eine mittelalterliche Literatur sichert, noch vor dem Ende des 18. Jahrhunderts das gesellschaftliche Interesse gefunden, das die wirtschaftliche Basis der Spielbücher seit dem 17. Jahrhundert formte. Dementsprechend haben wir nur sehr verstreute Belege. Der aufwendig geschnitzte Einbecker Spielstein hat kein Gegenstück; wahrscheinlich imitiert er Spielkarten. Ebenso zeigt das Amsterdamer Spiel eine Form, die sich auf Papier einfacher realisieren ließe. Aber solche Überlegungen können auch in die Irre führen. Die anderen frühen Spielsteinfunde aus England sind einfachste Knochenschnitzereien, und es lässt sich nicht einmal sagen, ob halbkugelige Aushöhlungen oder Rillen die ursprünglicherere Augenform sind. Letztlich ist die Zahl der Belege so gering, dass man nicht davon ausgehen darf, überhaupt einen repräsentativen Überblick über das frühe Formenspektrum zu besitzen. Dass Domino nicht in den frühen Spielbüchern auftaucht, ist allerdings ein eindeutiges Votum, dass die literate Elite kein Interesse an dem Spiel hatte. Vermutlich galt es eher als Kinderspiel oder Unterhaltung der einfachen Leute, denn auf der Mary Rose werden sicherlich keine Kinder gespielt haben.

Im letzten Drittel des 18. Jahrhunderts ist es jedenfalls in Frankreich und Deutschland gleichermaßen bekannt. Bemerkenswert ist allerdings, dass die frühen französischen Quellen deutlich renommierter sind als die deutschen. Sie zeigen nämlich ein bis in höchste Kreise akzeptables Gesellschaftsspiel, das häufig und mit Leidenschaft und Sachverstand (und um Geld) gespielt wurde. Die deutschen Quellen hingegen handeln von einem bedeutungsarmen Kinderspiel. Das ändert sich hier erst kurz nach Ende der napoleonischen Besetzung 1815. Seine Reputation fügt sich damit in den Kulturfluss von Frankreich nach Europa im Gefolge der militärischen Eroberungen ein, den 
man in der Kunstgeschichte als Empirezeit bezeichnet. Dieses Bild wird von dem Augsburger Kupferstich nur scheinbar gestört. Hier spielen zwar zwei hochgestellte Personen, aber man muss das ironisch-sexuelle Moment der Szenerie in Rechnung stellen. Das Haid ein belangloses Spiel anstatt eines anspruchsvollen wie Schach zeigt, verstärkt mindestens den Eindruck, dass es sich nur um einen Vorwand handelt. Möglicherweise ist die Wahl eines Kinderspiels sogar unmittelbar als sexuelle Anspielung zu verstehen.

Die Angabe des Wiener Anonymus, dass erst das vergrößerte Spiel mit 45 Steinen zur Beliebtheit des Domino geführt hätte, ist allerdings nicht nachzuvollziehen. Zwar ist es an sich eine sinnvolle Annahme, dass das Spiel in Gastwirtschaften größere Spielsätze gefördert hätte, aber in allen anderen Spielbüchern wird der Standardspielsatz mit 28 Steinen vorausgesetzt und andere Größen allenfalls am Rande erwähnt. Entweder gelten seine Angaben nur für Österreich, oder er will seinen Lesern eine private Vorliebe unterjubeln. Überhaupt darf man Angaben auch in gedruckten Quellen nicht zu unkritisch gegenüber treten, denn wenn Steine als „pariser Art" bezeichnet werden oder das Spiel in Norddeutschland unpopulärer sein soll als in Süddeutschland, können auch Marketinggesichtspunkte bzw. mangelnde Materialkenntnis die Ursache solcher Urteile sein.

Die Etymologie des Spiels hat viel Verwirrung gestiftet. Selbst in den frühesten Quellen gibt es offenbar keine einheitliche Erinnerung mehr an die Namensbedeutung. Da Derivate von dominus („Herr") in den Sprachen des lateinischen Westens weit verbreitet sind, ist es auch nicht schwer, spekulative Analogien zu konstruieren. Die Schwierigkeit mit der Ableitung von dem katholischen Priestermantel - direkt oder über den Umweg des Ausrufs im Piquet - liegt darin, dass dies auf den Schwarz-Weiß-Kontrast ebenholzverblendeter Beinspielsteine anspielt. Aber Domino ist auch im Französischen und Englischen ursprünglich die Bezeichnung des gesamten Spiels und eben gerade nicht des einzelnen Spielsteins so wie heute. Aber selbst wenn man diese Erklärung für möglich hält, so handelt es sich um eine bloße visuelle Analogie. Völlig verfehlt sind niedliche Phantasien wie der spielversessene Abt aus der Wikipedia. Wenn man mal von dem Oxforder Fund absieht, sind die Dominoquellen so weltlich wie nur irgend möglich. Von einer religiösen Beladung ist da schier überhaupt nichts zu erkennen.

Auch wenn der letzte Beweis dafür fehlt, so ist es doch recht wahrscheinlich, dass sich der Name von der historischen französischen Bezeichnung für Buntpapier ableitet. Dass im 18. und frühen 19. Jahrhundert Dominos als Spielkarten einigermaßen geläufig waren, lässt sich aus den Quellen gut nachvollziehen. Dass sich ein französischer Name international verbreitet, wäre kaum erstaunlich. Wir fassen ja erst die Bezeichnung, unter der sich das Spiel 
von Frankreich ausgehend international seine Literaturfähigkeit verdiente, in einer Schicht, die üblicherweise des Französischen mächtig war. Spätere Autoren haben dann natürlich das Problem, dass sie das altertümliche Wort gar nicht mehr kennen. Die Formulierung „Domino sein" ist vermutlich eine Volksetymologie. „Einen Domino machen" vertrüge sich als Bekundung der Vollendung auch durchaus noch mit der ursprünglichen Bedeutung, etwa im Sinne von „fertig sein“ und dann auch als isolierter Ausruf „Domino!“ Zudem kann man an den Beschreibungen von Gutsmuths und Catel auch nachvollziehen, dass der Begriff Domino sogar gebraucht wurde, um andere papierne Legespiele zu erläutern. Das Legen von graphischen Strukturen ist offenbar das semantisch entscheidende Element für den Namensgebrauch, weniger die Würfelanalogie.

Der Geltung des Domino als reputables Wettkampfspiel um Geld dürfte auch die Fertigung mit Ebenholzverblendungen attraktiv gemacht haben, wie sie bereits in der Anleitung in der Académie universelle des jeux beschrieben wird. Schwarze Verblendungen an Beinspielsteinen dienten wohl hauptsächlich dazu, der Individualisierung der Steine durch Schmutzwolken entgegen zu wirken. Dass sie dann auch nicht so leicht umfallen, wenn man sie auf dem Tisch aufstellt (Grunfeld und Oker, 1976, S. 104) ist ein angenehmer Nebeneffekt, aber sicher nicht die Ursache, weil man dafür auch helle Hölzer hätte verwenden können. Immerhin sind die frühen Beinspielsteine tatsächlich ziemlich dünn.

Ingram Braun

Hamburger Str. 25

D-34246 Vellmar

Deutschland

me@ingram-braun.net

https://ingram-braun.net/

Board Game Studies Journal 10, pp. 61-100 DOI $10.1515 /$ bgs-2016-0004 


\section{Literatur}

Art UK (o.J.). A Chelsea Pensioner Playing Dominoes. http:// artuk.org/discover/artworks/a-chelsea-pensioner-playing -dominoes-179489/ (besucht am 21. 3. 2016).

Auler, J. und Hiller, P. (2015). Buchweizen und Ziegenmilch. In Kronsbein, S. und Siepen, M., Herausgeber, Beiträge zur Archäologie des Niederrheins und Westfalens, Seiten 37-57. Verlag Stefan Kronsbein, Krefeld.

Bekkering, G. (2005). Deutsche Bilder aus dem 18. und 19. Jahrhundert auf Puzzlespielen. In Pieske, C., Vanja, K., Lorenz, D., und Nagy, S., Herausgeber, Tagungsband Ittingen 2004, Nummer 9 in Arbeitskreis Bild Druck Papier, Seiten 71-85. Waxman, Münster.

Bell, R. C. (1979). Board and Table Games from Many Civilizations. Dover Publications, Inc., New York.

Bestelmeier, G. H. (1979). Magazin von verschiedenen Kunst- und anderen nützlichen Sachen, zur lehrreichen und angenehmen Unterhaltung der Jugend, als auch für Liebhaber der Künste und Wissenschaften, welche Stücke meistens vorräthig zu finden bei G.H. Bestelmeier in Nürnberg. Edition Olms, Zürich.

Borst, A. (1986). Das mittelalterliche Zahlenkampfspiel. Nummer 5 in Supplemente zu den Sitzungsberichten der Heidelberger Akademie der Wissenschaften, phil.-hist. Kl. Carl Winter, Heidelberg.

Braun, I. (o.J.). Der archäologische Fund eines Dominosteins aus DormagenStürzelberg, Rhein-Kreis Neuss. Eingereicht.

Buxton Museum and Art Gallery (o.J.). A French Prisoner of War Cribbage and Dominoes set carved from bone. https:// enlightenmentderbyshire.wordpress.com/2012/07/25/a-french -prisoner-of-war-cribbage-and-dominoes-set-carved-from-bone/ (besucht am 23. 12. 2015).

Cambry (1803). Description du département de l'Oise, Band 2. P. Didot l'Aine, Paris.

Catel, P. F. (1790). Mathematisches und physikalisches Kunst-Cabinet, dem Unterrichte und der Belustigung der Jugend gewidmet. Lagarde und Friedrich, Berlin und Libau. 
Crawford Art Gallery, Cork (2014). http://www.crawfordartgallery.ie/ pages/paintings/FrankBramley.html (besucht am 14. 2. 2016).

Culin, S. (1895). Chinese Games with Dice and Dominoes. In Annual Report of the U.S. National Museum for 1893, Seiten 491-537. Government Printing Office, Washington.

Cunradi, J. H., Herausgeber (1810). Italienisch-deutsches und deutschitalienisches Waaren-Lexikon. Verlag der Steinischen Buchhandlung, Nürnberg.

Davies, P., Crook, P., und Murray, T. (2013). An Archaeology of Institutional Confinement. Nummer 4 in Studies in Australasian Historical Archaeology. Sydney University Press, Sydney.

Depaulis, T. (2007). Farbenspiel. In Schädler, U., Herausgeber, Spiele der Menschheit, Seiten 72-81. Wissenschaftliche Buchgesellschaft, Darmstadt.

Dixon, K. J. (2005). Boomtown Saloons. University of Nevada Press, Reno.

Drosdowski, G. und Grebe, P., Herausgeber (1963). Duden Etymologie. Nummer 7 in Der große Duden. Bibliographisches Institut, Mannheim u. a.

Dubosc, G. (1927). Le Jeu de dominos en Normandie. In Par ci, par là, Band 5, Seiten 47-58. Henri Defontaine, Rouen.

Dupuy, P. (1829). Abrégé élémentaire des differences les plus remarquables entre la France et l'Espagne. Joaquin Verdaguer, Barcelona.

Endrei, W. (1988). Spiele und Unterhaltung im alten Europa. Dausien, Hanau.

F. C. H. (1869). The Game of Dominoes. Notes and Queries, 4(3):80-81.

Fairclough, G. J. (1979). St. Andrews Street 1976. Nummer 2 in Plymouth Museum Archaeological Series. Plymouth City Museum and Art Gallery, Plymouth.

Furstner, H. (1985). Geschichte des niederländischen Buchhandels. Nummer 2 in Geschichte des Buchhandels. Otto Harrasowitz, Wiesbaden.

Gibson, E. S. (2009). Games. In Praetzellis, M. und Praetzellis, A., Herausgeber, South of Market: Historical Archaeology of 3 San Francisco Neighborhoods, Band 2, Seiten 319-320. California Department of Transportation, Oakland, California. 
Glonnegger, E. (1988). Das Spiele-Buch. Otto Maier, Ravensburg.

Grosley, P.-J. (1813). Euvres inédites, Band 2. C.-F. Patris, Paris.

Grunfeld, F. V. und Oker, E. (1976). Spiele der Welt. Wolfgang Krüger Verlag GmbH, Frankfurt am Main.

Gutsmuths, J. C. F. (1796). Spiele zur Uebung und Erholung des Körpers und des Geistes, für die Jugend, ihre Erzieher und alle Freunde unschuldiger Jugendfreuden. Verlag der Buchhandlung der Erziehungsanstalt, Schnepfenthal, 2. Auflage.

Heege, A. (1998). Zwischen Stift und Stadt. Archäologie in Niedersachsen, Seiten $86-87$.

Heege, A. und Roth-Heege, E. (2002). Einbeck im Mittelalter. Nummer 17 in Studien zur Einbecker Geschichte. Isensee, Oldenburg.

Heraldik-Wiki (2015). Dominostein (Heraldik). https://heraldik -wiki.de/index.php?title=Dominostein_(Heraldik) (besucht am 7. 3. 2016).

Herold und Wahlstab (1821). Bey Herold und Wahlstab in Lüneburg sind in diesem Jahre erschienen. Intelligenzblatt der Jenaischen Allgemeinen Literatur-Zeitung, (60):477-478.

Hesse, J. H. G. (1815). Bilder-Quodlibet in alphabetischer Ordnung. Magazin für Industrie und Literatur, Leipzig.

Hoffmann, D. (1983). Die Welt der Spielkarte. Edition Leipzig, Leipzig, 2. Auflage.

Hoffmann, D. (1995). Kultur- und Kunstgeschichte der Spielkarte. Jonas Verlag, Marburg.

Hoffmann, D. (1998). Die Anfänge im 15. und 16. Jahrhundert. Nummer 1 in Schweizer Spielkarten. Museum zu Allerheiligen Schaffhausen und Cartophilia Helvetica, Schaffhausen.

Holme, R. H. (1921). Dominoes. Notes and Queries, 12(9):447-448.

Hooper, J. (1882). „Domino“ as used by Omnibus Conductors. Notes and Queries, 6(5):229.

Jones, C. (1803). Hoyle's Games Improved. R. Baldwin, London. 
Jones, C. (1814). Hoyle's Games Improved. W. Lowndes, London.

Jørgensen, S. A., Herausgeber (1802). Nyeste dansk Spillebog. J. H. Schubothes, Kiobenhavn, 2. Auflage.

Kluge, K. E. (1860). Handbuch der Edelsteinkunde für Mineralogen, Steinschneider und Juweliere. F. A. Brockhaus, Leipzig.

Kretzschmer, J. C. (1838). Soldaten-, Kriegs- und Lagerleben, Band 2. L.-G. Homann, Danzig.

Krünitz, J. G. (1791). Oekonomisch-technologische Encyklopädie oder allgemeines System der Stats-Stadt-Haus- und Land-Wirthschaft, und der Kunst-Geschichte in alphabetischer Ordnung, Band 55. Joachim Pauli, Berlin.

Krünitz, J. G. und Korth, J. W. D. (1833). Ökonomisch-technologische Encyklopädie oder allgemeines System der Staats-, Stadt,- Haus-, und Landwirthschaft, und der Kunstgeschichte in alphabetischer Ordnung, Band 158. Joachim Pauli, Berlin.

Lambrick, G. und Woods, H. (1976). Excavations on the Second Site of the Dominican Priory, Oxford. Oxoniensa, 41:168-231.

Levy, D. (2013). What was the Hoyle copyright worth? (part 1). http://edmondhoyle.blogspot.com/2013/10/what-was-hoyle -copyright-worth-part-1.html (besucht am 30. 9. 2015).

Lhôte, J.-M. (1994). Histoire de jeux de societé. Flammarion, Paris.

Lhôte, J.-M. (2007). Wendezeit der Spielkultur. In Schädler, U., Herausgeber, Spiele der Menschheit, Seiten 114-127. Wissenschaftliche Buchgesellschaft, Darmstadt.

Lindauer, J. (о.J.). Bey Heinrich Friedrich Müller, Kunsthändler in Wien, am Kohlmarkt Nr. 1150 sind folgende neue Spiele erschienen, und bey Joseph Lindauer, Buchhändler in München zu haben. http://www.mdz-nbn-resolving.de/urn/resolver.pl?urn=urn: nbn: de:bvb:12-bsb11002233-6 (besucht am 7. 10. 2015).

Lo, A. (2000). The Game of Leaves. Bulletin of the School of Oriental and African Studies, 63(3):389-406.

Lo, A. (2004). China's Passion for Pai. In Mackenzie, C. und Finkel, I., Herausgeber, Asian Games, Seiten 216-231. Asia Society. 
Miller, B. (2015). Iron Age pottery, Victorian tin toys and butchered bone. http://www.culture24.org.uk/history-and-heritage/archaeology/ art528387-iron-age-pottery-victorian-tin-toys-and-butchered -bone-six-archaeological-finds-in-eastern-england-this-year (besucht am 17. 3. 2015).

Millot (1802). [Werbeanzeige]. Diario del Madrid, Seite 1047.

Mohl, M. (1845). Aus den gewerbswissenschaftlichen Ergebnissen einer Reise in Frankreich. J. G. Cotta, Stuttgart und Tübingen.

Morehead, A. H. und Mott-Smith, G. (1983). Hoyle's Rules of Games. The New American Library, New York, 2. Auflage.

Museum Victoria (o.J.). Domino - Bone, circa 1880 (Damaged). http:// collections.museumvictoria.com.au/items/1684726 (besucht am 23. 12. 2015).

Mytum, H. und Hall, N. (2013). Norman Cross: Designing and Operating an Eighteenth Century British Prisoner of War Camp. In Mytum, H. und Carr, G., Herausgeber, Prisoners of War, Nummer 1 in Contributions to Global Historical Archaeology, Seiten 75-91. Springer, New York.

N. N. (1680). The Compleat Gamester. Henry Brome, London, 2. Auflage.

N. N. (1694). Dictionnaire de L'Académie française, Band 1. Jean Baptiste Coignard, Paris.

N. N. (1718). Académie universelle des jeux. Le Gras, Paris.

N. N. (1762). Arts. L'Avantcoureur, Seiten 139-143.

N. N. (1770). Modes et nouveautes de Paris. Les Fastes du gout, ou les Nouveautés du jour, (1):119-121.

N. N. (1771a). Dictionnaire universel françois et latin, vulgairement appelé Dictionnaire de Trévoux, Band 3. La compagnie des libraires associés, Paris, 6. Auflage.

N. N. (1771b). Vocabulaire françois ou Abrégé du dictionnaire de l'Académie françoise, Band 1. Regnard \& Demonville, Paris.

N. N. (1780). Règles et principes sur le jeu de domino, avec les décisions des meilleurs joueurs. Fournier, Amsterdam und Paris. 
N. N. (1786). Académie universelle des jeux, Band 3. D. J. Changuion und T. Van Harrevelt, Amsterdam.

N. N. (1806). Academia dos Jogos, que trata do Voltarete, do Mediator, do Whist, do Boston, do Brelan, do Cassino, da Banca, das Damas, do Xadres, do Dominó, do Gamão, do Passo de Roma, e de outros muitos Jogos de Cartas e de Dados, Band 1. Impressão Regia, Lisboa.

N. N. (1808). Das neue Königliche L'Hombre nebst einer gründlichen Anweisung ... Herold und Wahlstab, Lüneburg, 15. Auflage.

N. N. (1815). Conversations-Lexikon oder encyclopädisches Handwörterbuch für gebildete Stände, Band 3. F. A. Brockhaus, Leipzig und Altenburg, 3. Auflage.

N. N. (1819). Archiv der Spiele oder fortlaufende Beschreibung der Vorwelt und Mitwelt, Band 1. Wilhelm Wittich, Berlin.

N. N. (1820). Il giuoco pratico o sieno. Sassi, Bologna.

N. N. (1825a). Il giuocatore nella sala di conversazione che istruisce. Vincenzo Abele Bussola, Milano, 3. Auflage.

N. N. (1825b). Pariser Sittentafel: Die Domino-Spieler. Der Sammler, 16(103):410-412.

N. N. (1828). Theoretisch-praktische Anleitung zu dem beliebten DominoSpiele, wie solches der strengen Räson gemäß, nach bestehenden Regeln, behandelt werden müsse, um den möglichst günstigen Erfolg zu sichern. Kaulfuß und Krammer, Wien.

N. N. (1829). Neuestes allgemeines Spielbuch. C. Haas, Wien.

N. N. (1838). Nürnberger Waaren-Kunde, oder Preisverzeichniss der vorzüglichsten Nürnberger Manufactur-Waren mit Angabe der Sorten, Grössen und Nummerirungen. C. Leuchs \& Comp., Nürnberg, 2. Auflage.

N. N. (1841). Annuaire général du commerce, de l'industrie, de la magistrature et de l'administration, ou almanach des 500,000 adresses. Firmin Didot frères, Paris.

N. N. (1846a). Dictionnaire de la conversation et de la lecture, Band 59. Garnier fréres, Paris. 
N. N. (1846b). Taschenbuch aller Karten-, Kegel-, Brett- und Würfel-Spiele. Herold und Wahlstab, Lüneburg, 18. Auflage.

N. N. (1855). The Encyclopcedia Britannica or Dictionary of Arts, Sciences and General Literature, Band 8. Adam und Charles Black, Edinburgh, 8. Auflage.

N. N. (1865). Allgemeine deutsche Real-Encyklopädie für die gebildeten Stände, Band 5. F. A. Brockhaus, Leipzig, 11. Auflage.

N. N. (1880). Henry VIII: Privy Purse Expences. In Gairdner, J., Herausgeber, 1531-1532, Nummer 5 in Letters and Papers, Foreign and Domestic, Henry VIII, Seiten 747-762. Her Majesty's Stationery Office, London.

N. N. (1910). The Encyclopcedia Britannica, Band 8. University Press, Cambridge, 11. Auflage.

Nicolai, F. (1795). Beschreibung einer Reise durch Deutschland und die Schweiz, im Jahre 1781, Band 9. Carl Ernst Bohn, Berlin und Stettin.

Oettermann, S. (1997). The Panorama. Zone Books, New York.

P. F. y P. (1839). Juegos de la Ciudadela, del Asalto ó ma Ataque y Defensa. Juan Francisco Pifferer, Barcelona.

Perth Museum and Art Gallery (o.J.). French prisoner of war dominoes. http://www.pkc.gov.uk/article/6624/French-prisoner-of -war-dominoes (besucht am 23. 12. 2015).

Platt, C. und Coleman-Smith, R. (1975). The finds. Nummer 2 in Excavations in Medieval Southampton 1953-1969. Leicester University Press, Leicester.

Redknap, M. (2005). Recreation: Games and Gaming. In Gardiner, J. und Allen, M. J., Herausgeber, Before the Mast, Nummer 4 in The Archaeology of the Mary Rose, Seiten 133-141. Cromwell Press, Trowbridge.

Rodriguez, B. J., Herausgeber (1845). Diccionario universal francés-español, Band 2. R. J. Dominguez, Madrid.

Sanchez, C. (2014). Le livres des jeux aux XVII et XVIII e siècles. Diplomarbeit, Université Lumière Lyon II, Lyon.

Savary des Bruslons, J. und Savary, P. L. (1723). Dictionnaire universel de commerce, Band 1. Jacques Estienne, Paris. 
Schmid, A. (1847). Literatur des Schachspiels. Carl Gerold, Wien.

Schreiber, W. L. (1937). Die ältesten Spielkarten und die auf das Kartenspiel Bezug habenden Urkunden des 14. und 15. Jahrhunderts. J. H. Ed. Heitz, Straßburg.

Seymour, R. (1734). The Compleat Gamester. E. Curll und J. Wilford, London, 5. Auflage.

Southampton City Council (2014). Archaeology Object Database. http:// www. southampton.gov.uk/archaeology/search.asp (besucht am 14. 3. 2016).

Stauss, T. (2015). Frühe Spielwelten. Librum, Hochwald.

Strutt, J. (1801). Glig-gamena angel-deod, or, The sports and pastimes of the people of England. J. White, London.

Teuber, S. (2009). Einbeck-Petersilienwasser. Nummer 41 in Materialhefte zur Ur- und Frühgeschichte Niedersachsens. Marie Leidorf, Rahden/Westf.

The George Washington Foundation (o.J.). Archaeology at Kenmore. http://www.kenmore.org/kenmore/kp_arch.html (besucht am 23. 12. 2015).

Thümmler, S. (1998). Die Geschichte der Tapete. Edition Minerva, Eurasburg.

Tobler, T. (1837). Appenzellischer Sprachschatz. Orell, Füßli und Compagnie, Zürich.

University of Iowa Office of the State Archaeologist (o.J.). The Fort Atkinson Story. http://archaeology.uiowa.edu/files/archaeology.uiowa .edu/files/OSA_FtAtkinsonTourGuide\%20for\%20web.pdf (besucht am 23. 12. 2015).

Van Braam-Houckgeest, A. E. (1798). Voyage de l'ambassade de la Compagnie des Indes Orientales hollandaises vers l'empereur de la Chine, dans les années 1794 \& 1795, Band 2. L'Editeur, Philadelphia.

Van Braam-Houckgeest, A. E. (1799). The Games and Public Amusements of the Chinese. The Monthly Magazine, 8:594-595.

van Delft, P. und Botermans, J. (2004). Denkspiele der Welt. Weltbild, Augsburg. 
van der Linde, A. (1874). Geschichte und Literatur des Schachspiels. Julius Springer, Berlin.

van der Linde, A. (1881). Das erste Jahrtausend der Schachlitteratur (8501880). Julius Springer, Berlin.

Vegas, A., Herausgeber (1815). Diccionario geografico universal, que comprehende la description de las quatro partes del mundo, Band 4. Francisco Martinez d'Ávila, Madrid.

von Düben, C. G. F. (1819). Talisman des Glücks oder der Selbstlehrer für alle Karten-, Schach-, Billard-, Ball-, und Kegel-Spiele. C. G. Flittner, Berlin, 2. Auflage.

von Düben, C. G. F. (1820). Neueste Anleitung zur gründlichen Erlernung des gewöhnlichen neuen Billard-, Kegel-, Ball-, Damen- und sogenannten polnischen Damen- und Dominospiels. C. G. Flittner, Berlin, 5. Auflage.

von Keeß, S. und Blumenbach, W. C. W. (1829). Systematische Darstellung der neuesten Fortschritte in den Gewerben und Manufacturen und des gegenwärtigen Zustandes derselben, Band 1. Carl Gerold, Wien.

von Salis, U. (1776). Philanthropinischer Erziehungsplan oder vollständige Nachricht von dem ersten wirklichen Philanthropin zu Marschlins. Eichenberg, Frankfurt am Mayn.

von Salis, U. (1777). Plan van philanthropynsche opvoeding of volledig bericht van het eerste philantropinum te Marschlins. H. A. de Chalmot und D. Romar, Leeuwarden und Franeker.

Watts, P. M., Herausgeber (1829). The New Year's Gift and Juvenile Souvenir. Longman $\mathrm{u}$. a., London und Philadelphia.

Weekley, E. (1911). Dominoes: their Origin. Notes and Queries, 11(3):345346.

Weekley, E. (1921). An etymological dictionary of modern English. John Murray, London.

Wessex Archaeology (2010a). Death and Dominoes - The First POW Camp (Norman Cross, Cambridgeshire). http://www.wessexarch.co.uk/ blogs/time-team/2011/04/18/time-team-series-17-death-and -dominoes-first-pow-camp-norman-cross-cambri (besucht am 5. 10. 2015). 
Wessex Archaeology (2010b). Norman Cross Camp Cambrigdeshire. http://www. wessexarch.co.uk/system/files/71507_TT\%20Norman\% 20Cross.pdf (besucht am 5. 10. 2015).

White, A. (2001). Two objects made by French Prisoners-of-War in Lancaster City Museum. Contrebis, 26:32-34.

Wikipedia (o.J.). Domino. https://de.wikipedia.org/wiki/Domino (besucht am 4. 10. 2015).

Williams, N. (1971). Henry VIII and his court. Weidenfeld \& Nicolson, London.

Willshire, W. H. (1876). A descriptive catalogue of playing and other cards in the British Museum accompanied by a concise general history of the subject and remarks on cards of divination and of a politico-historical character. Trustees, London.

Wittmann, R. (1982). Der lesende Landmann. In Buchmarkt und Lektüre im 18. und 19. Jahrhundert, Nummer 6 in Studien und Texte zur Sozialgeschichte der Literatur, Seiten 1-45. Max Niemeyer, Tübingen.

Wittmann, R. (2011). Geschichte des deutschen Buchhandels. C. H. Beck, München, 3. Auflage.

Zangs, C. und Holländer, H., Herausgeber (1994). Mit Glück und Verstand. Thouet, Aachen.

Zimmermann (1778). [Werbeanzeige]. Hannoversche Anzeigen von allerhand Sachen, deren Bekanntmachung dem gemeinen Wesen nöthig und nützlich sind, Seiten 1605-1606. 